\title{
LA DINÁMICA DE LA VOLATILIDAD DEL IPC Y SUS COMPONENTES
}

\author{
Jorge Ludlow Wiechers*
}

Departamento de Economía

Universidad Autónoma Metropolitana-Atzcapotzalco

Beatríz Mota Aragón

Departamento de Economía

Universidad Autónoma Metropolitana-Iztapalapa

(Recibido 28 de abril 2005, aceptado 9 de junio 2005)

\section{Resumen}

En este trabajo modelamos la volatilidad de los rendimientos del IPC y sus componentes en los últimos años (agosto 1998, julio 2005), hay interés en los años 2003-2004 pues reportaron importantes rendimientos para la BMV. Para explicar la relación riesgo rendimiento partimos de la hipótesis de mercados eficientes (HME) y de los modelos de equilibrio. Después utilizamos el estadístico BDS $(m, \varepsilon)$ para demostrar que las series usadas son no-lineales. A partir de aquí analizamos el riesgo con una especificación no-lineal de volatilidad multivariada. El modelo multivariado MV-GARCH(1,1). Los niveles de riesgo de los índices sectoriales y del IPC mostrados de mayor a menor riesgo son: Extractiva, Comunicaciones, Comercio, Servicios, IPC, Varios, Construcción, Transformación.

\section{Abstract}

In this work we model the volatility of the yield and its components of the financial index, called IPC, along August 1998 to July 2005, special focus is set in the period 2003-2004 because the Mexican market had returns above normal. We start with the efficient market hypothesis and equilibrium models. We use the BDS test to see whether the series are non-linear, thus a non linear specification is required to model a multivariate dynamics, the model used is the MV-GARCH(1,1). Is shown that the sectors become ordered as: Mining, Communications, Commerce, Services, IPC, Various, Building, Transformation, being the first the more risky and the last variable the less one.

Clasificación JEL: C32, C87, G11, G14

Palabras clave: Índice de mercado, Riesgo-Rendimiento, Varianza condicional, Volatilidad multivariada, Modelos GARCH

* Departamento de Economía, División CSH, Universidad Autónoma Metropolitana-Atzcapotzalco. Av. San Pablo 180, Col. Reynosa Tamaulipas, c.p. 02200, México D.F. Teléfonos: $+52(55) 53189424$ y $+52(55) 53189136$ ext. 112. Correos electrónicos: macroescenarios@yahoo.com.mx; jlw@correo.azc.uam.mx y bma@xanum.uam.mx 


\section{Introducción}

La internacionalización y apertura del sistema financiero mexicano iniciada en la década de los noventa ha arrojado cambios con consecuencias radicales en los diferentes sectores que lo conforman, particularmente el mercado bursátil ha logrado un desarrollo pujante en la captación y colocación de recursos en los últimos años, tal como lo muestra el IPC de la BMV. Específicamente en los años (2003-2004) el crecimiento del indicador marca un comportamiento altamente significativo. Esta razón nos lleva a estudiar y modelar la volatilidad de los rendimientos del IPC y sus componentes en este período, los cuales reportaron importantes rendimientos para la bolsa de valores.

Partimos de la teoría financiera de mercados de capital y de manera específica de la hipótesis de mercados eficientes (HME) y de los modelos de equilibrio. Después demostramos utilizando el estadístico $\operatorname{BDS}(m, \varepsilon)$ que la serie IPC, no es una serie IID sino que el comportamiento de los rendimientos del IPC es no-lineal. A partir de aquí analizamos el riesgo y por tanto la volatilidad con un MV-GARCH $(1,1)$. Por ser un modelo multivariado se presenta la covolatilidad, con varianza condicional tiempo dependiente, propuesta por Engle y Kroner (1995), a partir de un trabajo, de Baba Y, R. Engle, D, Kraft, y K. Kroner (1987) conocida como la formulación BEKK para capturar este enlace dinámico.

El Índice de Precios y Cotizaciones de la Bolsa Mexicana de Valores (IPC) como sabemos, mide la rentabilidad del comportamiento del mercado accionario. La muestra se integra actualmente de 35 emisoras de distintos sectores de la economía y representa el total de los títulos accionarios cotizados en el mercado bursátil. Las variaciones porcentuales del índice, reflejan el aumento o la disminución del nivel medio de los precios de la muestra accionaria, esto es, reflejan día a día el resultado de una jornada de actividades; cuando es un mercado pujante este índice crece, pero cuando el mercado esta flojo el índice desciende. Con 1725 días de mercado operados desde el lunes 21 de agosto de 1998 hasta el viernes 29 de julio de 2005 . Vamos a medir el movimiento de los índices sectoriales en relación al IPC.

El rendimiento del IPC se mide por el cambio porcentual usando la función logaritmo $r(t)=100^{*} \operatorname{Ln}[x(t) / x(t-1)]$. Debido a que se manejan 1725 días operados lo cual hace que si presentamos las gráficas por fechas resulten totalmente ilegibles, por lo que se decidió establecer un consecutivo entre las fechas y los números en las gráficas, que se detalla en el anexo I, (21 de diciembre 1998 es el día 1, 2 enero 2003 día 1054, día 2 enero 2004 día 1315, 2 enero 2005 día 1598 y 29 de julio 2005 es el día 1725 ). Las gráficas de los rendimientos de los índices IPC y de los sectores de la actividad económica se muestran a continuación.

Al revisar los histogramas de las series de cada uno de los indicadores sectoriales en estudio, observamos que la volatilidad cambia en el tiempo y que tiende a agruparse (clusters), esto es, un cambio grande en la volatilidad es persistente (hay un periodo de incertidumbre). 
Gráfica 1.A IPC.

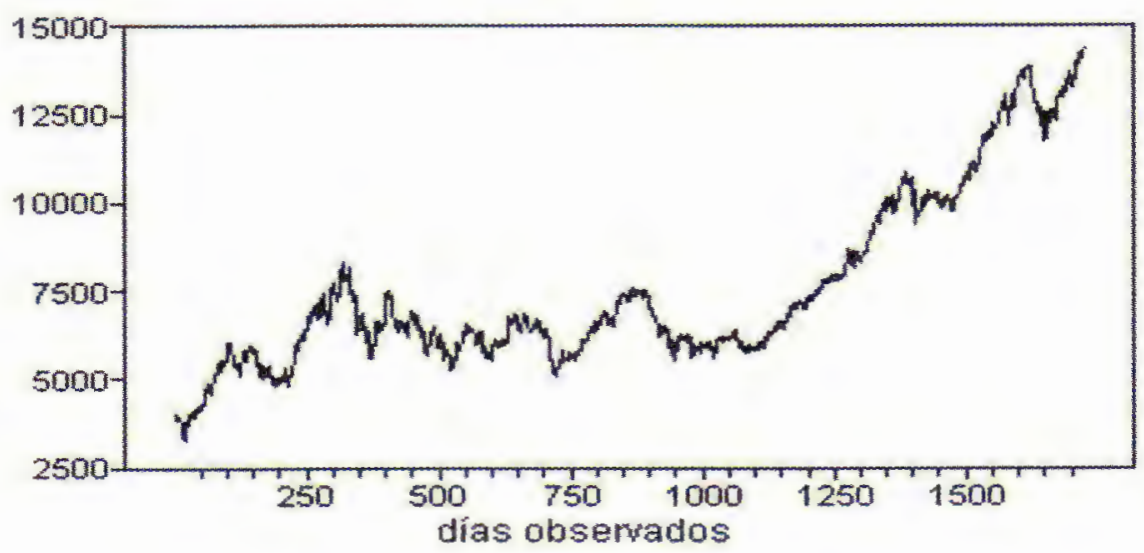

Gráfica 1.B Rendimiento IPC.

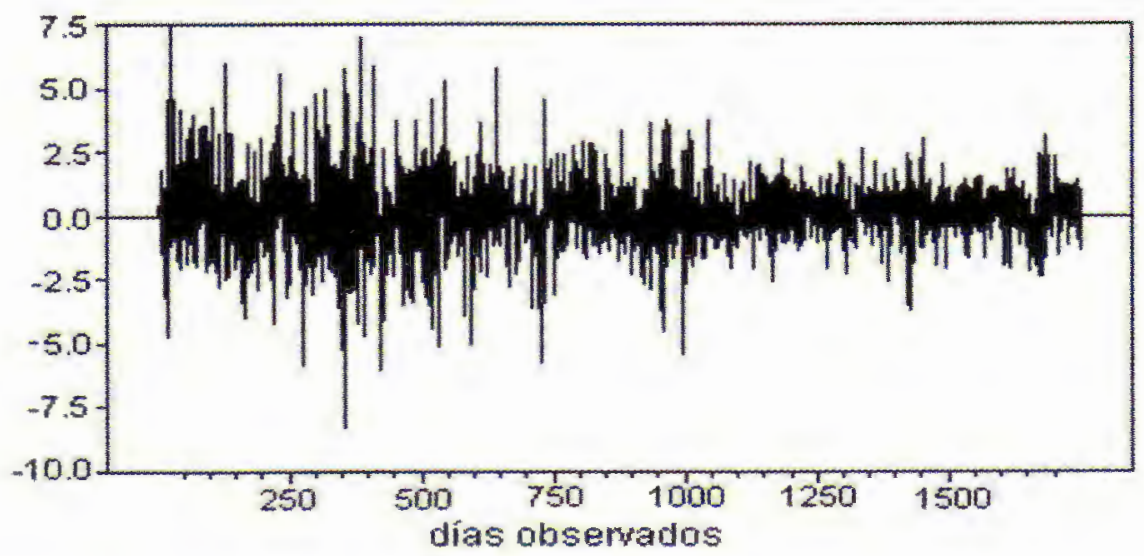

Gráfica 1.C Extractiva.

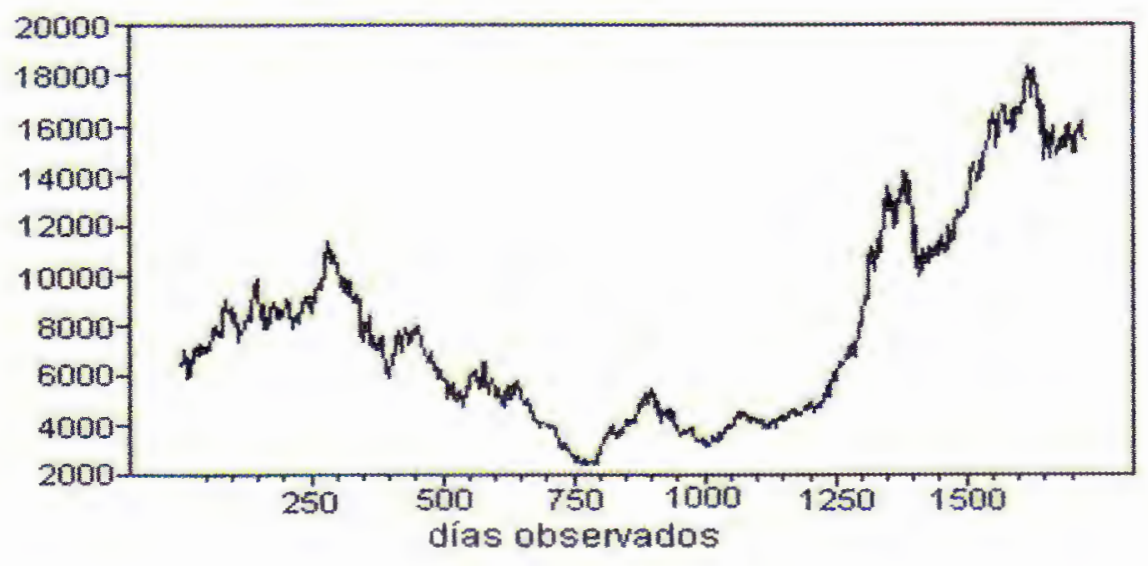


Gráfica 1.D Rendimiento Extractiva.

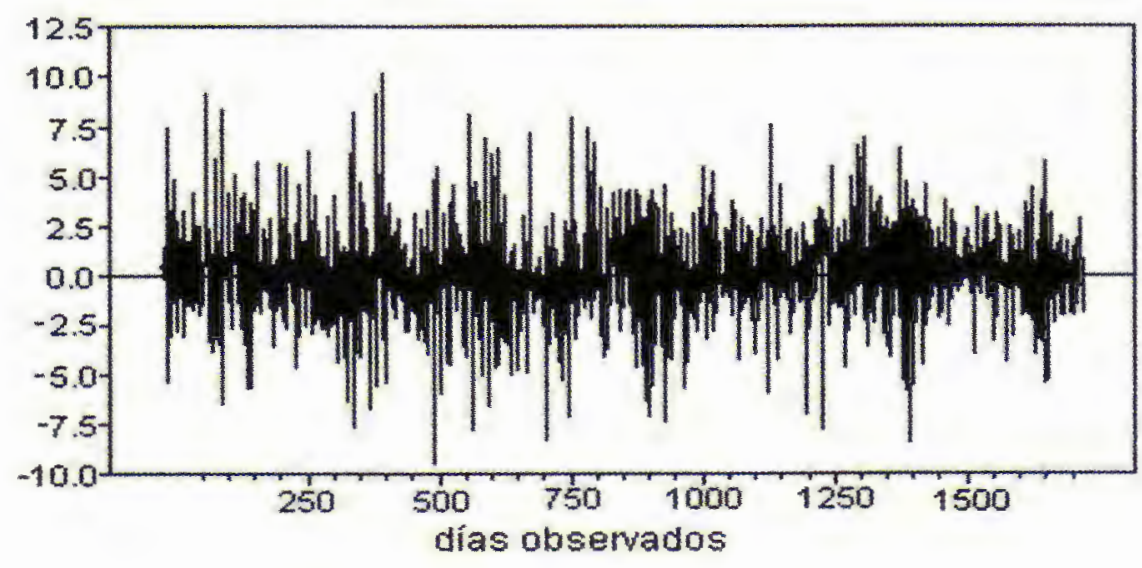

Gráfica 1.E Transformación.

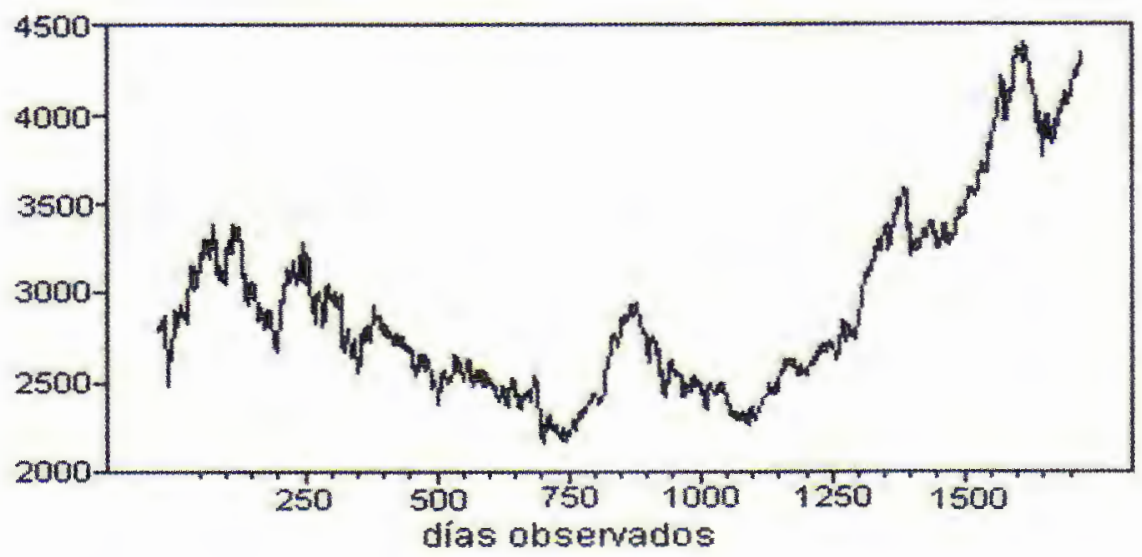

Gráfica 1.F Rendimiento Transformación.

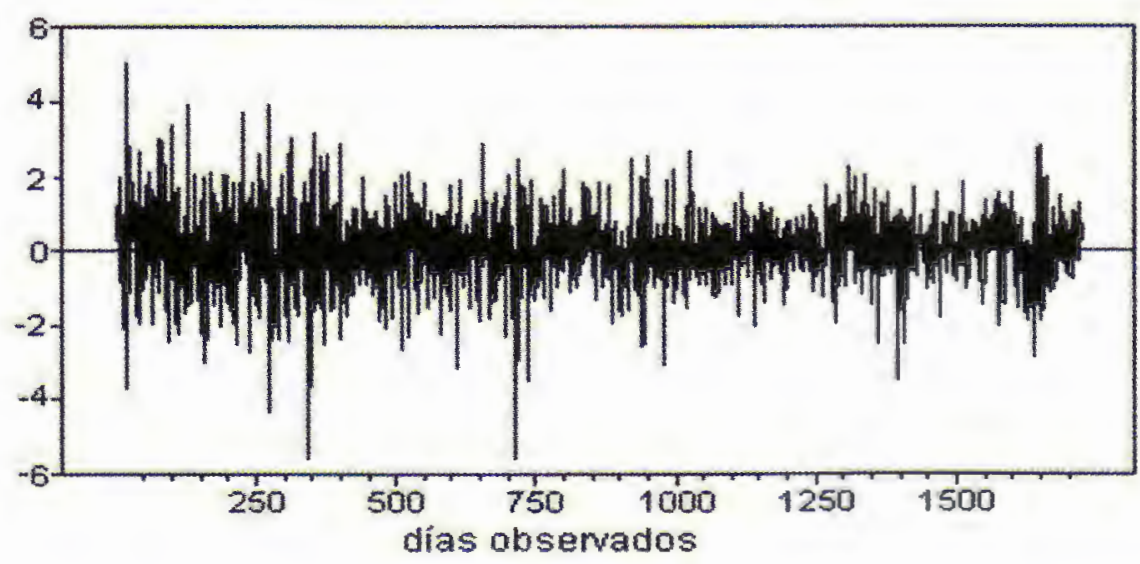


Gráfica 1.G Construcción.

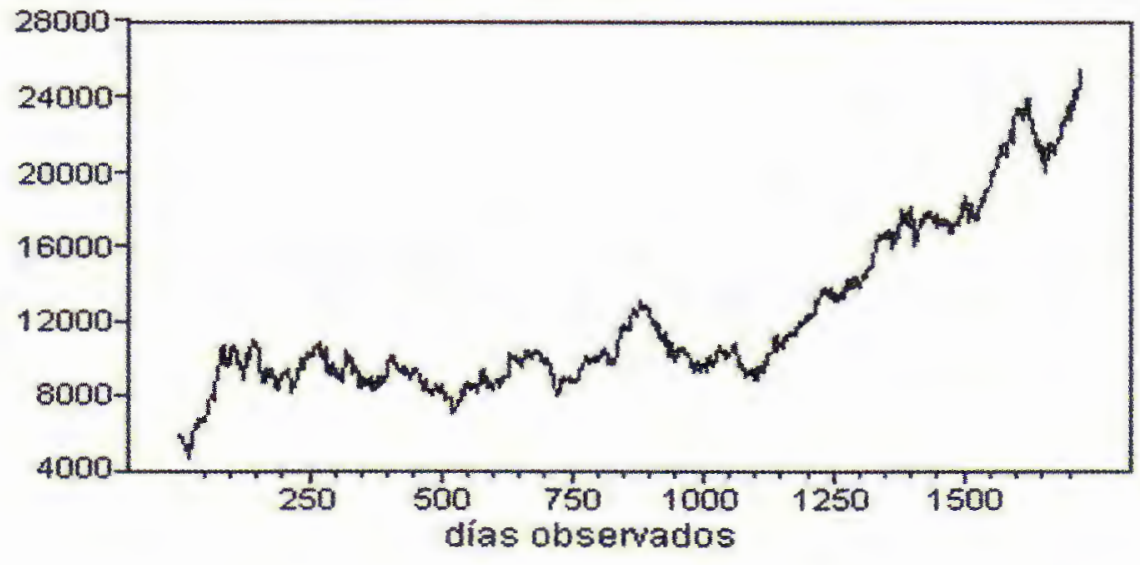

Gráfica 1.H Rendimiento Construcción.

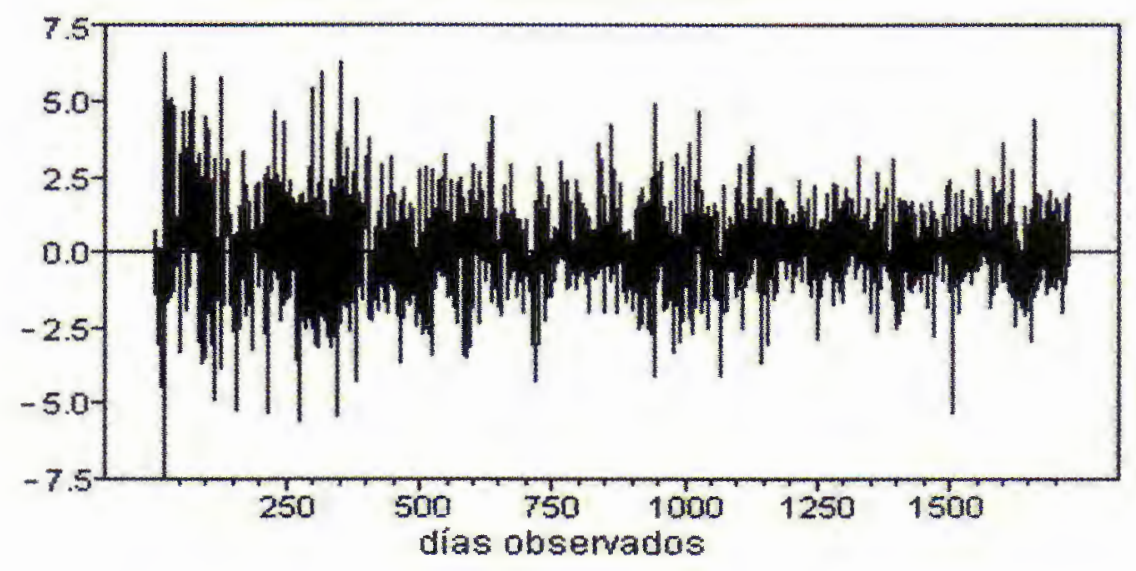

Gráfica 1.I Comercio.

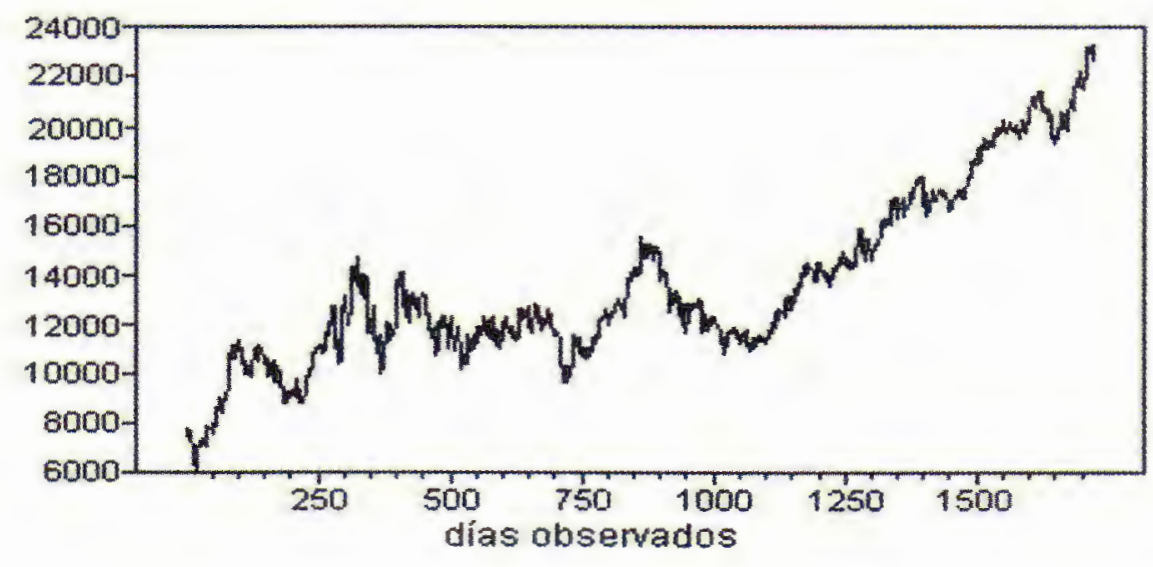


Gráfica 1.J Rendimiento Comercio.

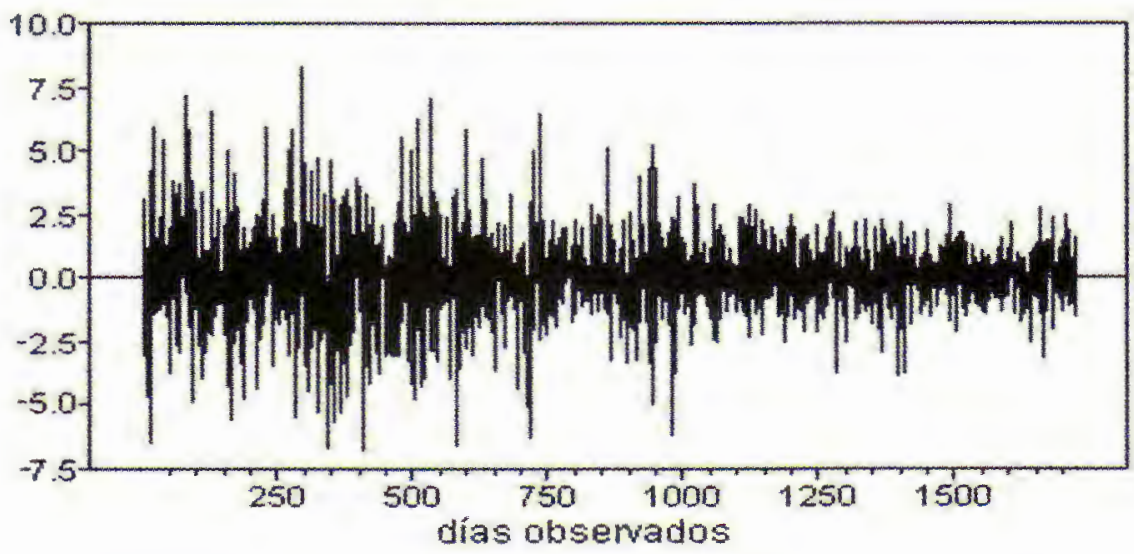

Gráfica 1.K Comunicaciones.

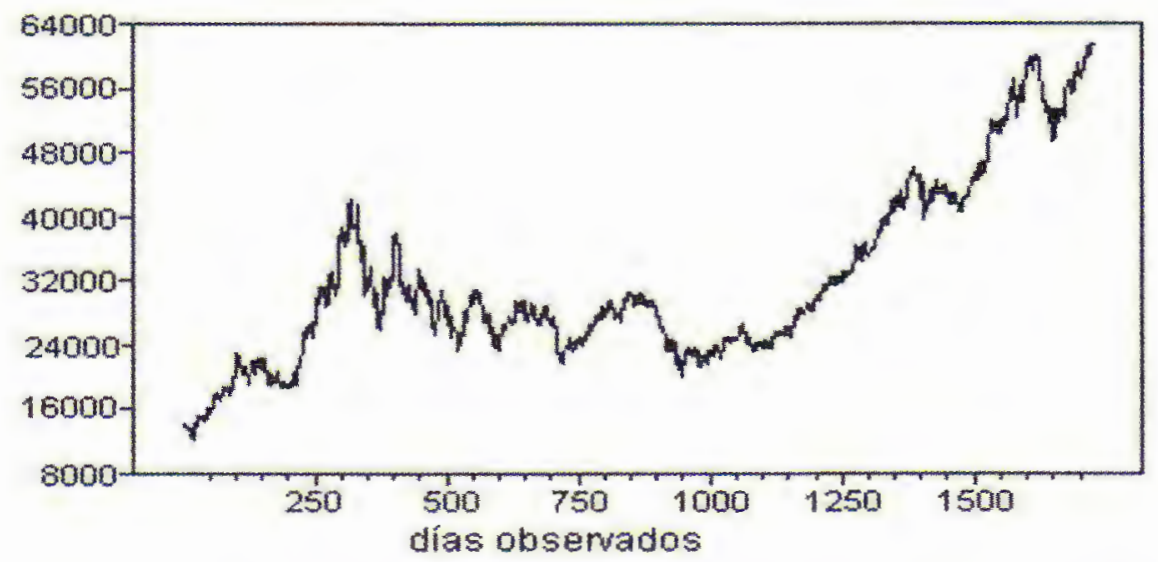

Gráfica 1.L Rendimiento Comunicaciones.

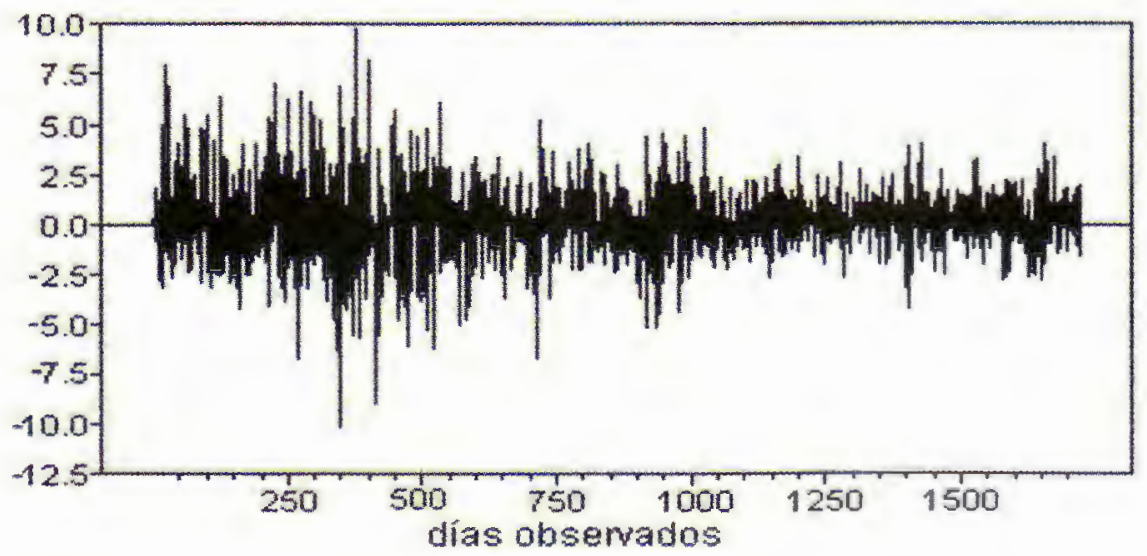


Gráfica 1.M Servicios.

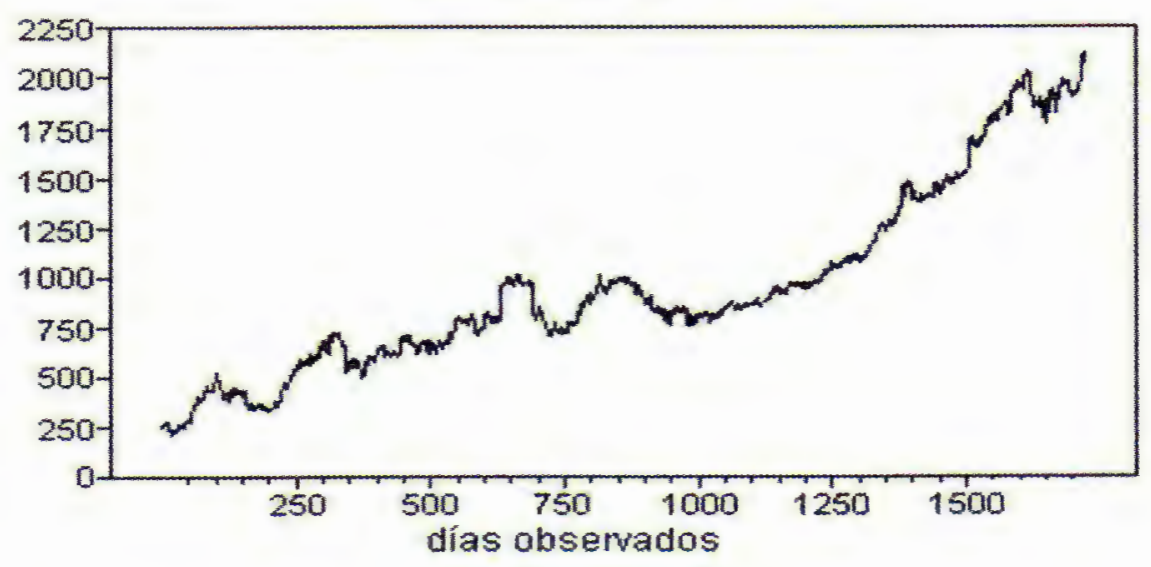

Gráfica 1.N Rendimiento Servicios.

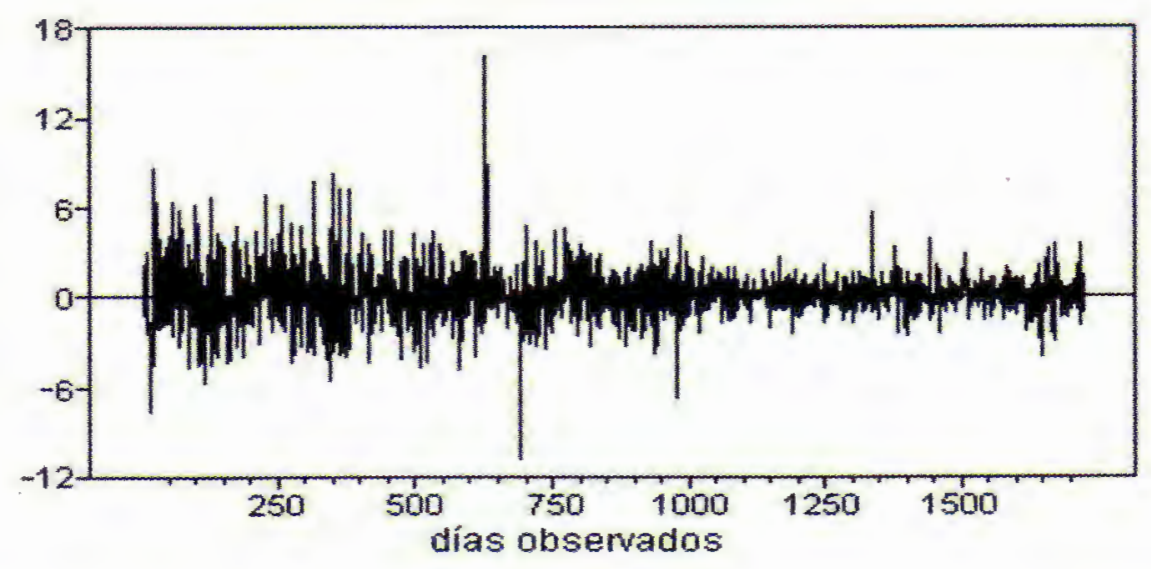

Gráfica 1.0 Varios.

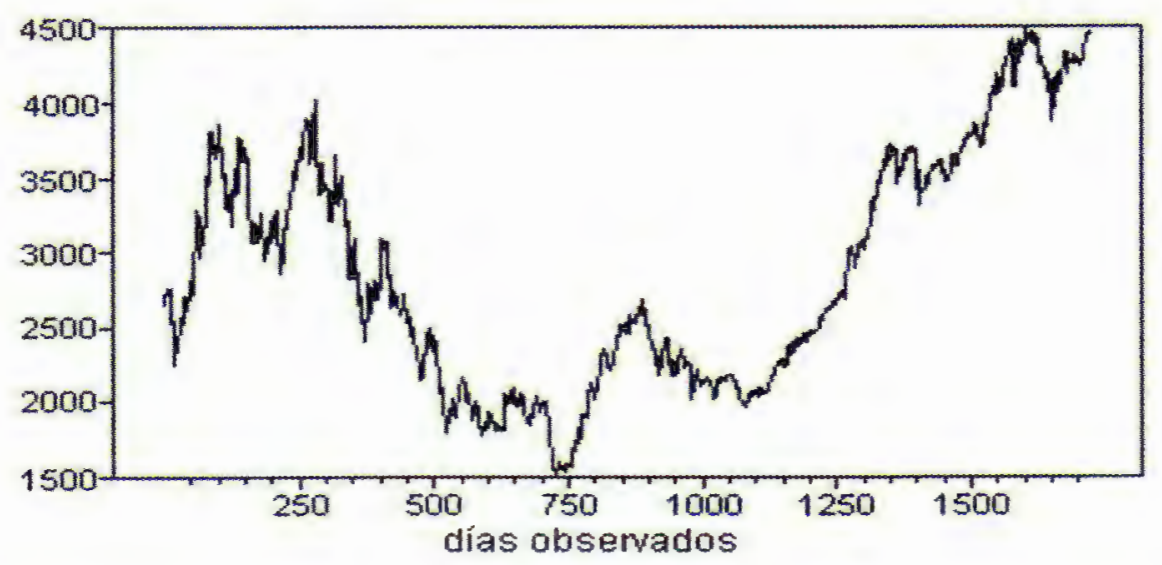




\section{Gráfica 1.P Rendimiento Varios.}

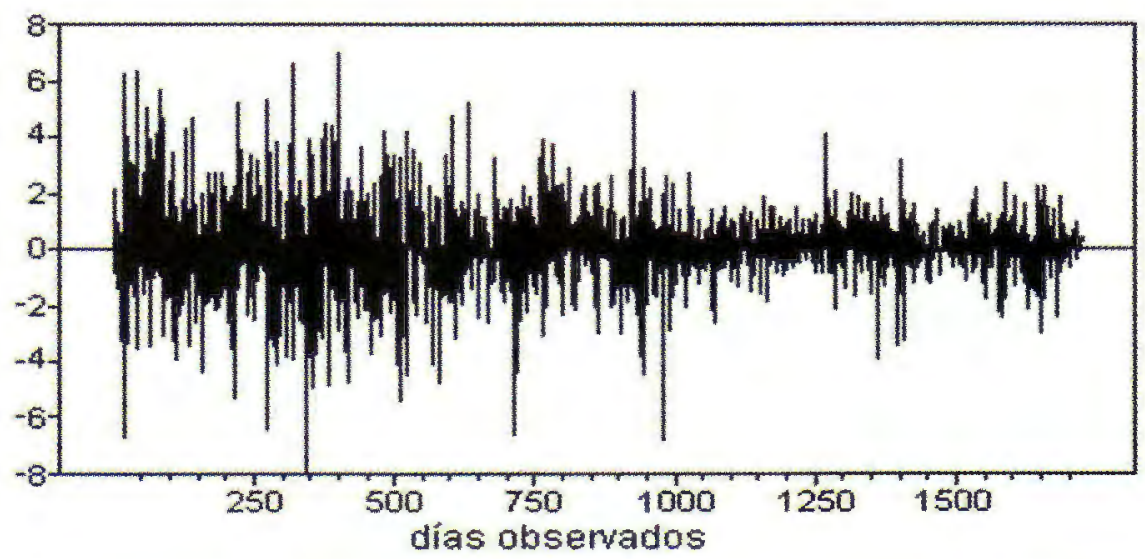

Podemos notar en las gráficas que el rendimiento sostenido esta asociado con una disminución en el riesgo, ya que la volatilidad de las gráficas de los rendimientos va siendo menor. Como es claro, el nivel de riesgo está relacionado con el nivel de rendimiento, por lo que se espera que los inversionistas tomen el nivel de riesgo que será compensado por el nivel de rendimiento esperado, es decir, un alto riesgo estará asociado con un alto rendimiento, de los modelos de equilibrio esto es equivalente a: $\mathrm{E}\left(R_{m}\right)-E\left(R_{0}\right)>0$.

La segunda sección de este trabajo abarca una exhaustiva revisión de teorías del mercado de capital con la finalidad de construir un marco teórico. La tercera describe la metodología a utilizar, la nuestra y los resultados de la investigación. La última sección plantea las conclusiones e indica algunas líneas de investigación futuras.

\section{Elaborando el marco para el análisis}

La investigación financiera ha generado un número importante de trabajos en esta dirección. Desde los modelos tradicionales de selección de cartera; Markowitz (1952, 1959), del CAMP; Sharpe (1964), Lintner (1965), el APT; Ross (1976a, 1976b), Black (1972), el modelo intertemporal (ICAMP); Merton (1973), el CAMP orientado al consumo; Breeden (1979). Estudios recientes de sección cruzada; Nicholson (1960), trabajos sobre anomalías; Basu (1977), Banz (1981). Investigaciones de los retornos reversibles de largo plazo; DeBondt y Thaler (1985), Chopra, Lakonishok y Ritter (1992), y sobre retornos de corto plazo; Jegadeesh (1990) y Jegadeesh y Titman (1993). De correlación en el corto plazo Fama y Frech (1992), hasta los trabajos de "dos y tres factores" (Fama y MacBeth (1973) y Fama y French (1993) respectivamente). Las implicaciones del modelo de dos factores para los rendimientos esperados se derivande la relación riesgo - rendimiento esperado para un activo y para una cartera. ${ }^{1}$

1 Esta relación se explica a partir de: $E\left(R_{i}\right)-E\left(R_{m}\right)=S_{m}\left[\sum_{j=1}^{N} X_{j m} \sigma_{i j} / \sigma\left(R_{i}\right)-\right.$ $\left.\sigma\left(R_{m}\right)\right]$. Básicamente la ecuación dice que la diferencia entre el retorno esperado sobre el activo y el retorno esperado sobre la cartera es proporcional a la diferencia entre el riesgo del activo y el riesgo de la cartera. 
Del modelo de tres factores se predice que dicho modelo explica la relación riesgo-rendimiento al incorporar algunas anomalías que el CAMP no considera. La aceptación que han tenido estos modelos puede verse en diversas aplicaciones, Quigley y Sinquefield (2000) y Davis (2001).

Pero vamos a analizar esto con más detalle, en el debate empírico no sólo esta dada la relación positiva riesgo-rendimiento, sino que es importante explicar los pronósticos de los rendimientos futuros y para ello se usa el análisis fundamental. (Fama (1991)) analiza las pruebas para pronosticar rendimientos y establece dos escenarios: el corto y el largo plazo, argumenta que es posible predecir los rendimientos futuros de corto plazo a través de los retornos pasados e incorpora en el precio de la acción otras variables de la empresa (dividendos, ingresos). Mientras que en el largo plazo incorpora variables estructurales. En el corto plazo (diario y fin de semana), los precios pasados resultaron insignificantes para predecir los precios futuros. Por el contrario, Jegadeesh y Titman (1993), observaron que los rendimientos en el corto plazo tienden a continuar; las acciones con altas ganancias en los 20 meses previos tienden a tener altos rendimientos en el futuro. Como veremos esto se cumple en nuestro estudio.

En el horizonte de corto plazo hay pequeñas autocorrelaciones, Roll (1986), Fama (1992). Fama y French (1988), mostraron evidencia de autocorrelación negativa en un horizonte de 3 a 5 años, sin embargo, mostraron que desaparece en un plazo mayor, tomando como referencia el período 1926-1940, finalmente concluyeron que los rendimientos esperados varían con el tiempo. Estas variaciones son consistentes con el modelo intertemporal del precio de los activos (ICAMP).

En el largo plazo, DeBondt y Thaler (1985), encontraron un comportamiento reversible en los rendimientos (acciones con bajos retornos en el pasado tienden a tener altos rendimientos en el futuro y viceversa) ya que el mercado sobrereacciona. ${ }^{2}$ Chopra, Lakonishok y Ritter (1992), mostraron que la tendencia de los retornos reversibles es otra contradicción del CAMP. De acuerdo con Summers (1986), en el horizonte de largo plazo el precio de las acciones toma una caída larga y suave.

Ahora bien, los rendimientos del índice accionario son explicados en Fama y Frech (1988), y Poterba y Summers (1988). En el largo plazo (2-10 años) el índice sigue la tendencia media-reversible en el tiempo. Esto sugiere una tendencia sistémica y que el nivel general de los rendimientos esperados dependa de las condiciones de las variables macroeconómicas (análisis fundamental). En este sentido, el índice debería ser un estimador razonable del comportamiento del mercado y de los fundamentales que son determinados racionalmente.

Existe evidencia empírica de la relación directa entre la actividad financiera y las variables macroeconómicas. Particularmente relación entre los retornos esperados de las acciones del mercado y dichas variables (Fama (1970, $1990 \mathrm{y}$ 1991)). Otros estudios (Chen, Roll y Ross (1986); Chan y Chen (1991) y Kwon et. al. (1997)), propusieron la relación del precio de las acciones y las tasas de interés, la inflación, la tasa de crecimiento (GNP, en inglés, PIB, en español), la tasa de desempleo, el índice de productividad, etc.

2 Veáse K. C. Chan and Nai-fu Chen (1991). Structural and Return characteristics of small and large firms. Journal of Finance, 46, 1467-1484. 
Otro punto de vista importante de la teoría financiera que soporta los estudios sobre el comportamiento de los rendimientos de las acciones son los trabajos desarrollados sobre procesos estocásticos de Markov, los cuales predicen que únicamente el valor presente de la variable es relevante para pronosticar el futuro. Los valores pasados y los presentes que se derivan del pasado son irrelevantes, por lo tanto los pronósticos futuros son inciertos y podrían expresarse en términos de distribuciones de probabilidad. Entonces, la propiedad de Markov implica que la distribución de la probabilidad del precio en algún punto en el futuro es independiente de los precios pasados.

La idea de eficiencia es una idea muy antigua en la teoría económica y fue retomada en Fama (1970) para describir el funcionamiento de los mercados financieros. ${ }^{3}$ El autor argumenta que los mercados son eficientes si los precios reflejan plenamente la información disponible, es decir, ajustándose de inmediato a la nueva información que llega al mercado, y que los cambios en los precios son aleatorios y los rendimientos en los precios son constantes. El modelo de Black-Sholes (1973), propone que el precio de las acciones siguen un paseo aleatorio. Implica una distribución normal en el corto plazo en los cambios proporcionales del precio de las acciones y también implica que el precio de las acciones en el futuro tiene una distribución lognormal.

Otras investigaciones mostraron que los sucesivos cambios en los precios de las acciones individuales son independientes, Fama et. al. (1969), Mandelbrot (1966) y Samuelson (1965), señalaron rigurosamente la independencia de los cambios sucesivos en los precios. Después de la la propuesta de Fama y MacBeth (1973), que prueba una relación positiva riesgo rendimiento a través del modelo teórico de cartera y de los modelos de equilibrio, han surgido otras investigaciones que argumentan que no es posible obtener rendimientos constantes a través de la información ya que los retornos esperados se relacionan con el nivel de riesgo de los activos, (Fama y French (1993)). Más tarde otros trabajos señalaron que es posible pronosticar el rendimiento esperado de las acciones, Fama (1991), Jegadeesh y Titman (1993), DeBondt y Thaler (1985).

El punto de partida para nuestro análisis es iniciar con la idea de mercados eficientes (Fama: los mercados son eficientes si los precios reflejan plenamente la información disponible, este criterio es adecuado, sin duda.), pero notando que la definición usa la idea de una caminata al azar, por lo que se basa en una condición lineal. Mientras que es reconocido que la estructura de un índice tipo IPC tendría que ser no-lineal, en cuyo caso la dinámica es compleja. Vamos a revisar esta idea con mucho mas cuidado.

\section{Metodología}

Primero vamos a aplicar el test $\operatorname{BDS}(m, \varepsilon)$ (Brock, Dechert, Scheinkman, Le Baron (1996)), para demostrar que el IPC es no-lineal. Esta es una prueba de propósito general para determinar el nivel de dependencia entre los datos (lo cual depende de la dimensión de correlación $m$, y de la distancia, entre los

3 Paul Samuelson (1965). Proof That Properly Anticipated Prices Fluctuase Randomly. Industrial Management Review, 41-49. Explica que los cambios en los precios son aleatorios y no son predecibles aún incorporando las expectativas e información del mercado. Retomado por E. Fama (1970). Efficient Capital Markets: A Review of Theory and Empirical Work. Journal of Finance, pp. 383-417. 
datos, no mayor a $\epsilon$ ), tiene como hipótesis nula que la serie esta conformada por una lista de variables independientes e idénticamente distribuidas (IID).

La prueba $\operatorname{BDS}(m, \varepsilon)$ es muy útil para mostrar la existencia de patrones que se observan en los datos comparándolo con lo que se debiera observar con datos IID. Uno debe estar atento a que el resultado en la prueba BDS depende de $l o s$ valores tomados en $m$ y $\varepsilon$. Con un valor grande (pequeño) de $\varepsilon$ la correlación entre los puntos tendera a ser elevada (baja). Mientras la dimensión de correlación $m$, sea mayor resulta que menor será el numero de trayectorias que no se traslapen y el valor del estadístico BDS será mayor.

Es importante notar que al ser una prueba de propósito general, hace que el rechazo de la hipotes nula, nos envíe como alternativa a alguno de los siguientes casos, aquí es donde se toman valores específicos de $m$ y $\varepsilon$. $\Lambda$ ) Se presenta dependencia lineal entre los datos. B) la serie no es estacionaria. C) Se presenta una dependencia no lineal, que puede llegar a denotar caos.

Bajo la hipótesis nula: se trata de variables IID, se espera que el estadístico BDS este cercano a cero en toda $m$. O sea, si generamos datos IID bajo la distribución normal, se debe obtener $\operatorname{BDS}(m, \varepsilon) \equiv 0$ en toda dimensión $m$. Visto de otra forma, el estadístico BDS rechaza la hipótesis nula cuando $Z-$ stat $>2(\mathrm{P}$-value $<0.01)$, por lo que se afirma que la serie analizada, no es una serie IID sino que es no-lineal. Variando el nivel de $m$, en $\operatorname{BDS}(m, 0.3)$ se obtiene la Tabla 1.

Tabla 1. Prueba BDS.

\begin{tabular}{ccccc} 
Dimension & BDS Stat. & Std. Error & z-Stat. & P-Value \\
\hline 2 & 0.195600 & 0.001546 & 126.5399 & 0.0000 \\
3 & 0.247769 & 0.001087 & 228.0183 & 0.0000 \\
4 & 0.257213 & 0.000575 & 447.2983 & 0.0000 \\
5 & 0.254236 & 0.000267 & 951.8209 & 0.0000 \\
6 & 0.247896 & 0.000115 & 2154.375 & 0.0000 \\
7 & 0.240879 & $4.72 \mathrm{E}-05$ & 5102.752 & 0.0000 \\
8 & 0.233925 & $1.87 \mathrm{E}-05$ & 12496.79 & 0.0000 \\
9 & 0.227190 & $7.24 \mathrm{E}-06$ & 31375.43 & 0.0000 \\
10 & 0.220690 & $2.75 \mathrm{E}-06$ & 80273.58 & 0.0000 \\
\hline
\end{tabular}

Como se puede leer en la tabla; BDS en las columnas $Z$-stat y en P-value sistemáticamente rechaza la idea de que se trate de una serie IID.

El resultado anterior es interesante porque nos motiva a buscar entender, ¿que hay en su dinámica no-lineal? A partir de las múltiples investigaciones derivadas en torno al análisis de la no linealidad en el comportamiento de las acciones, se ha incluido en la agenda de investigación el análisis detallado sobre la medición del riesgo y por tanto de la volatilidad de los mercados, para esto se ha desarrollado tecnología avanzada que ha ampliado la familia de los modelos GARCH y vamos a utilizarla para entender la BMV a través de revisar el comportamiento del IPC y los índices sectoriales.

La medición de la volatilidad se logra al desarrollarse las técnicas para la heterocedasticidad, ésta al inicio fue considerada como una falla en la especificación del modelo, posteriormente la filosofía en la construcción de modelos, 
gracias al análisis de residuos, hizo que en el programa de trabajo se íncorporara la idea de modelar la dinámica de los residuos por lo cual se comenzó a hablar de residuos $\operatorname{ARMA}(p, q)$ al terminar casi esta etapa Breusch, Pagan, Goffrey y otros, iniciaron un análisis para la heterocedasticidad y su estimación consistente, pero fue Robert Engle (1982), quien mostró como modelar la volatilidad condicionada tiempo dependiente a través de un proceso ARCH (AutoRegressive Conditional Heteroskedasticity). Es posible plantearse un modelo de regresión con errores $\mathrm{ARCH}(1)$ que modela la varianza de las series con base en las perturbaciones aleatorias pasadas este es:

$$
\begin{gathered}
Y_{t}=\beta^{\prime} \chi+\epsilon_{t}, \\
\sigma_{t}^{2}=\alpha_{0}+\alpha_{1} \epsilon_{t-1}^{2}, \quad \epsilon_{t} \mid \epsilon_{t-1} \approx N\left[0, \sigma_{t}^{2}\right] .
\end{gathered}
$$

$\mathrm{Si}\left|\alpha_{1}\right|<1$, entonces incondicionalmente,

$$
\epsilon_{t} \approx N\left[0, \alpha_{0} /\left(1-\alpha_{1}\right)\right] \text {. }
$$

Engle y Kraft (1983), han empleado el $\operatorname{ARCH}(q)$ más general,

$$
\epsilon_{t} \mid \epsilon_{t-1}, \epsilon_{t-2}, \cdots, \epsilon_{t-q} \approx N\left[0, \sigma_{t}^{2}\right] .
$$

Esto se acostumbra poner como: Siendo el conjunto de información $\Psi_{t-1}=$ gen $\left\{\epsilon_{t-1}, \epsilon_{t-2}, \cdots, \epsilon_{t-q}, \cdots\right\}$ que es el espacio generado por toda la historia de las innovaciones del proceso. $\epsilon_{t} \mid \Psi_{t-1} \approx N\left[0, \sigma_{t}^{2}\right]$, por lo que un $\operatorname{ARCH}(q)$ es:

$$
\sigma_{1}^{2}=\alpha_{0}+\alpha_{1} \epsilon_{t-1}^{2}+\alpha_{2} \epsilon_{t-2}^{2}+\cdots+\alpha_{q} \epsilon_{t-q}^{2}, \quad \alpha_{0}>0, \alpha_{1}>0, \alpha_{2}>0, \cdots, \alpha_{q}>0
$$

y

$$
\left|\alpha_{1}+\alpha_{2}+\cdots+\alpha_{q}\right|<1, \quad \epsilon_{t} \approx N\left[0, \alpha_{0} /\left(1-\alpha_{1}-\alpha_{2}-\cdots-\alpha_{q}\right)\right] .
$$

La varianza condicional tomando los residuos es:

$$
\sigma_{1}^{2}=\alpha_{0}+\alpha_{1} e_{t-1}^{2}+\alpha_{2} e_{t-2}^{2}+\cdots+\alpha_{q} e_{t-q}^{2} \quad \text { (se esta modificando) }
$$

mientras que la varianza NO-condicional es

$$
\alpha_{0} /\left(1-\alpha_{1}-\alpha_{2}-\cdots-\alpha_{q}\right) .
$$

Peters (2001), argumentó que se requieren procesos ARCH de orden elevado para capturar la dinámica de la volatilidad condicionada y Tim Bollerslev (1986), propuso el modelo ARCH generalizado (Generalized AutoRegressive Conditional Heteroskedasticity - GARCH). El cual se describe:

$$
\epsilon_{t} \mid \Psi_{t-1} \approx N\left[0, \sigma_{t}^{2}\right],
$$

donde la varianza condicional es

$$
\sigma_{t}^{2}=\alpha_{0}+\delta_{1} \sigma_{t-1}^{2}+\delta_{2} \sigma_{t-2}^{2}+\cdots+\delta_{p} \sigma_{t-p}^{2}+\alpha_{1} \epsilon_{t-1}^{2}+\alpha_{2} \epsilon_{t-2}^{2}+\cdots+\alpha_{q} \epsilon_{t-q}^{2} .
$$


Definimos

$$
Z_{t}=\left[1, \sigma_{t-1}^{2}, \sigma_{t-2}^{2}, \cdots, \sigma_{t-p}^{2}, \epsilon_{t-1}^{2}, \epsilon_{t-2}^{2}, \cdots, \epsilon_{t-q}^{2}\right]^{\prime}
$$

y

$$
\gamma=\left[\alpha_{0}, \delta_{1}, \delta_{2}, \cdots, \delta_{p}, \quad \alpha_{1}, \cdots, \alpha_{q}\right]^{\prime}=\left[\alpha_{0}, \delta^{\prime}, \alpha^{\prime \prime}\right]^{\prime}
$$

Entonces,

$$
\sigma_{t}^{2}=\gamma^{\prime} Z_{t}
$$

El GARCH(1,1) es un caso muy usado en la práctica.

$$
\varepsilon_{t}=\sqrt{h_{t}} \nu_{t}, \quad \nu_{t} \approx \text { iid } N(0,1) .
$$

Varianza condicional $h_{t}$ definida como:

$$
h_{t}=w+\alpha_{1} \varepsilon_{t-1}^{2}+\beta_{1} h_{t}
$$

donde

$$
w>0, \quad \alpha_{1}>0, \quad \beta_{1} \geq 0, \quad \alpha_{1}+\beta_{1}<1 .
$$

Un modelo que en el caso univariado es muy sencillo, es tomar como ecuación de regresión el rendimiento observado oscilando sobre su rendimiento esperado y con una innovación que sea un $\operatorname{GARCH}(1,1)$

$$
r_{t}=\mu+\epsilon_{t}, \quad \epsilon_{t} \sim \operatorname{GARCH}(1,1) .
$$

Más tarde se inicia una familia muy amplia de modelos con volatilidad multivariada, con la propuesta originalmente del modelo GARCH Multivariado, usando la formulación llamada Vech, Bollerslev, Engle y Woolridge (1988). La formulación propuesta por Engle y Kroner (1995), a partir de un trabajo de Baba, Engle, Kraft y Kroner (1987), conocida como la formulación BEKK es la que vamos a utilizar en el presente trabajo. Finalmente, Bollerslev (1990), propone la clase de modelos de correlación condicional constante.

Es claro, que la volatilidad de los activos financieros se modifica a lo largo del tiempo, por lo cual los modelos univariados son muy usados, pero además, se presenta una covolatilidad, (una liga de covarianzas entre los cuadrados de las innovaciones de los rendimientos), por lo que también se requiere de un modelo multivariado para capturar este enlace dinámico, el cambio en la volatilidad de los mercados debido a la covolatilidad. La formulación BEKK es flexible aunque el problema que esto conlleva es el número de parámetros a estimar, "Curse of Dimensionality" o sea, que hay demasiados parámetros.

MV-GARCH $(1,1)$ con varianza condicional tiempo dependiente, tiene la descomposición usual:

$$
\begin{gathered}
y_{t}=\mathrm{E}\left[y_{t} \mid \psi_{t-1}\right]+\varepsilon_{t}, \\
\operatorname{Var}\left[\varepsilon_{t} \mid \psi_{t-1}\right]=H_{t} .
\end{gathered}
$$

Donde $\psi_{t-1}$ es el conjunto de toda la información disponible hasta/incluso $t-1$, $H_{t}=\left(h_{i j t}\right)$ es una matriz simétrica y positiva definida excepto en un conjunto $\Omega$ tal que $\mathrm{P}[\Omega]=0 . H_{t}$ es $n \times n$ donde $n$ es el número de entradas del vector $\epsilon_{t}$. 
Explícitamente el modelo BEKK (Engle \& Kroner 1995), que se va a estimar es:

$$
\begin{gathered}
R_{t}=\mu+\varepsilon_{t}, \\
H_{t}=C^{\prime} C+A_{1}^{\prime} \varepsilon_{t} \varepsilon_{t} A_{1}+B_{1}^{\prime} H_{t-1} B_{1} .
\end{gathered}
$$

Como es usual se tienen los vectores columna correspondientes a los rendimientos $R_{t}$, las innovaciones $\varepsilon_{t}$, y la matriz de varianza covarianza $H_{t} . R_{t}=$ $\left(r_{i t}\right), \varepsilon_{t}=\left(\varepsilon_{i t}\right)$ y una matriz $H_{t}=\left(h_{i j t}\right)$ note que $C$ (simétrica), $A_{1}, B_{1}$ son matrices $n \times n, n$ es el número de entradas del vector $\varepsilon_{t}$, por lo que tenemos: 8 parámetros a estimar de $\mu, 36=8^{*}(8+1) / 2$ de la matriz simétrica $C, y$ por último $2^{*} 64=2^{*} 8^{*} 8$ parámetros para $A_{1}$ y $B_{1}$.

Se aplica el modelo BEKK con $172=8+36+2^{*} 64$ parámetros a estimar para las 8 variables con 1725 días de mercado operados desde el 21 de agosto de 1998 hasta el 29 de julio de 2005 . Una vez obtenidas las estimaciones consistentes e insesgadas debido a que se usa el método de máxima verosimilitud para realizar la estimación, se pueden obtener las varianzas condicionales simultáneas las cuales tienen por gráficas:

Gráfica 2.A IPC varianza condicional.

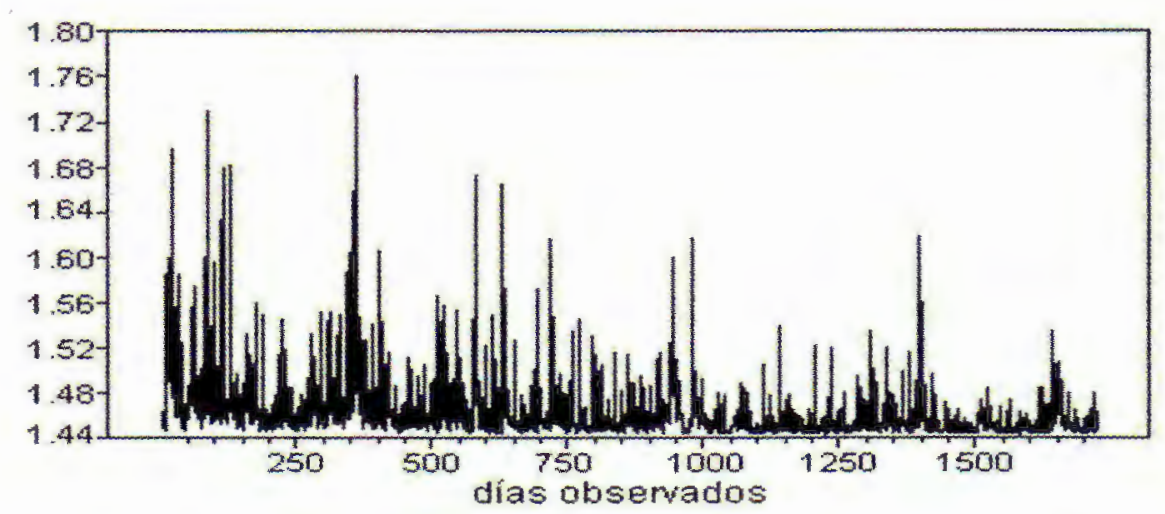

Gráfica 2.B Extractiva varianza condicional.

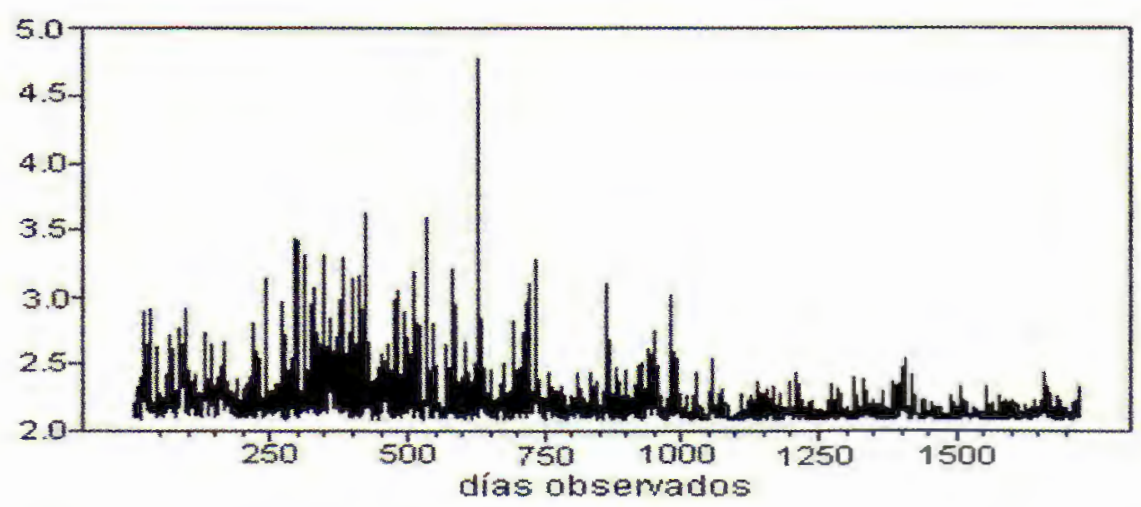




\section{Gráfica 2.C Transformación varianza condicional.}

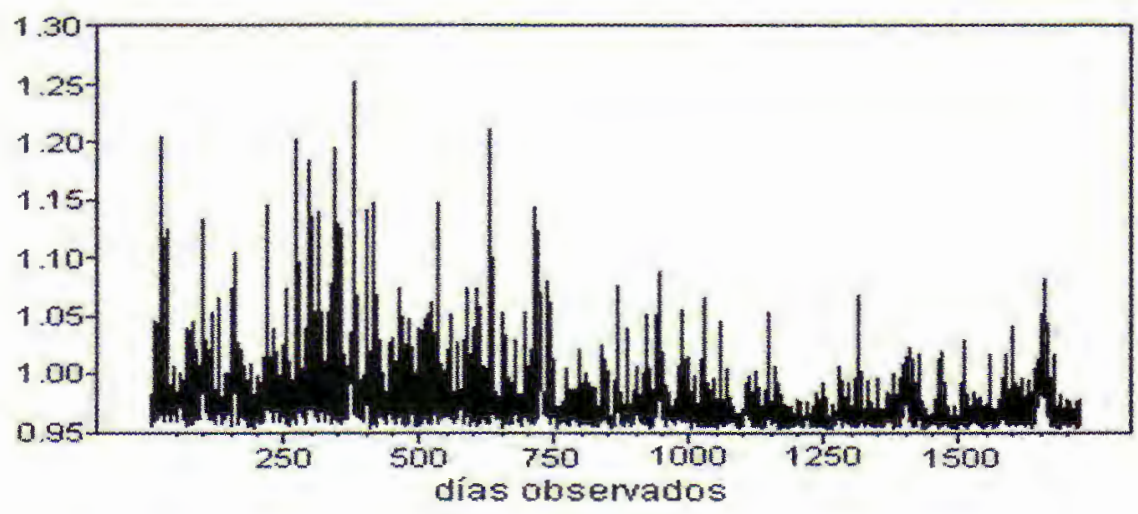

Gráfica 2.D Construcción varianza condicional.

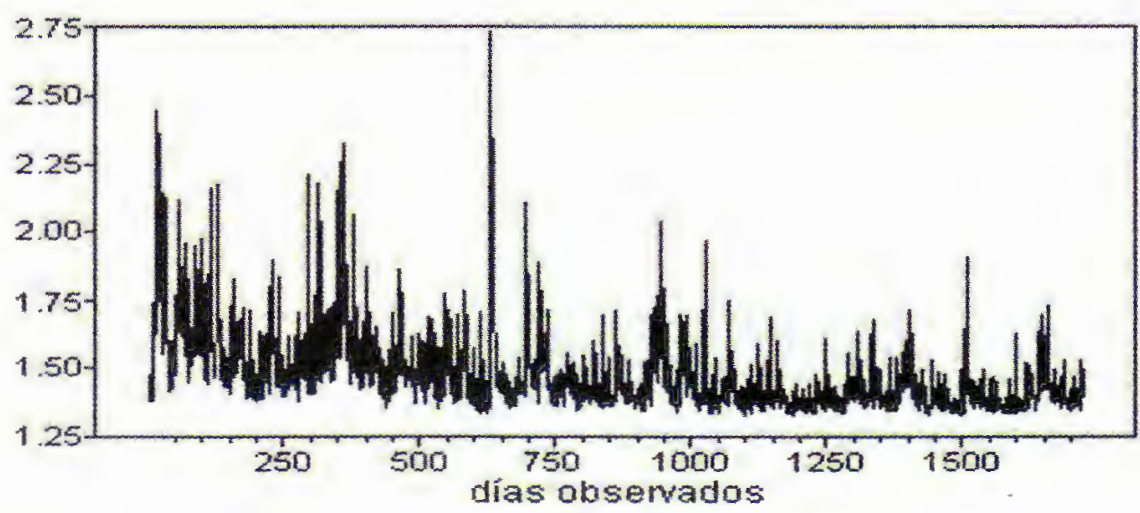

Gráfica 2.E Comercio varianza condicional.

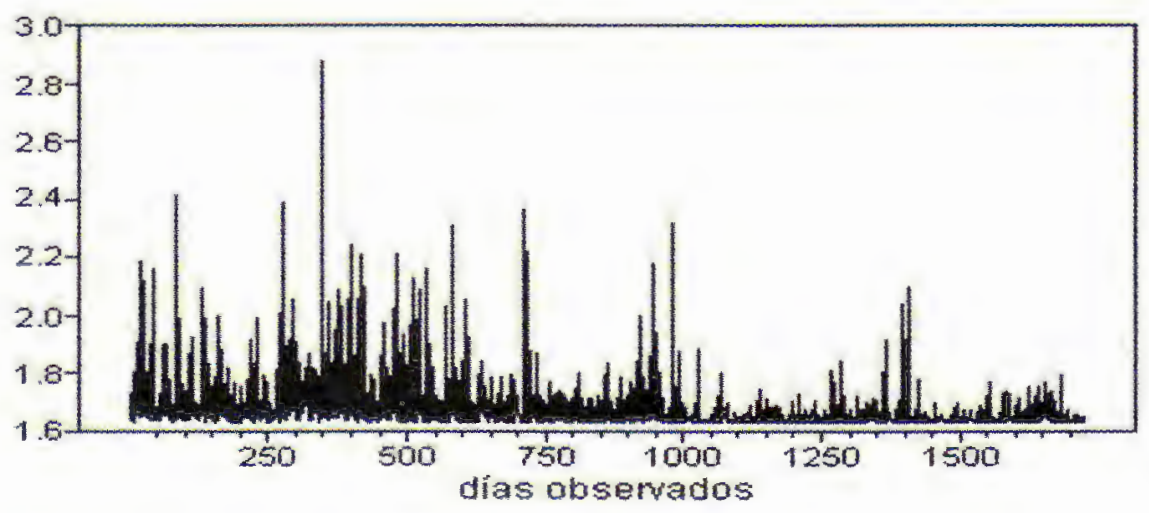


Gráfica 2.F Comunicaciones varianza condicional.

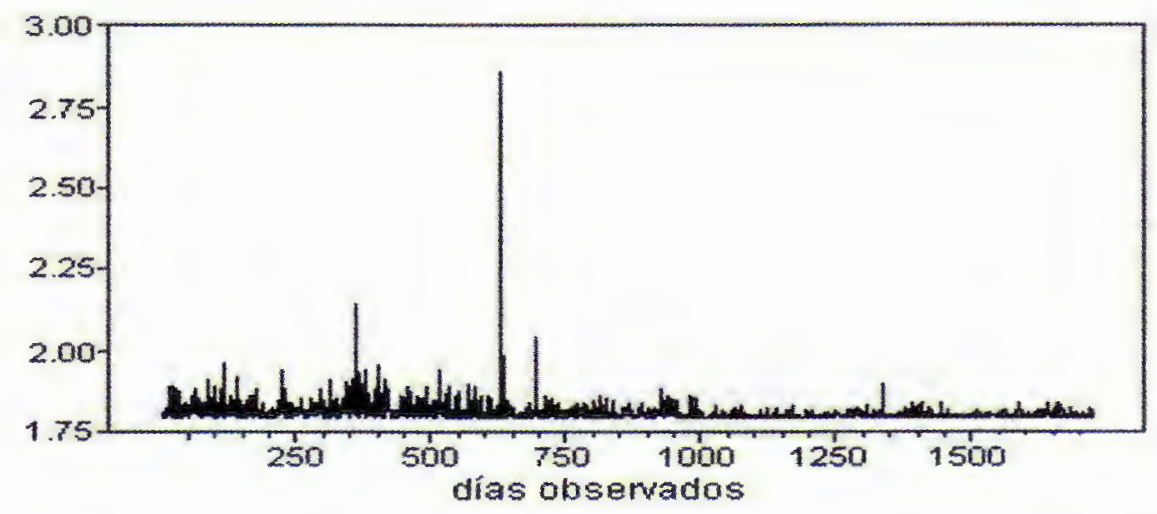

Gráfica 2.G Servicios varianza condicional.

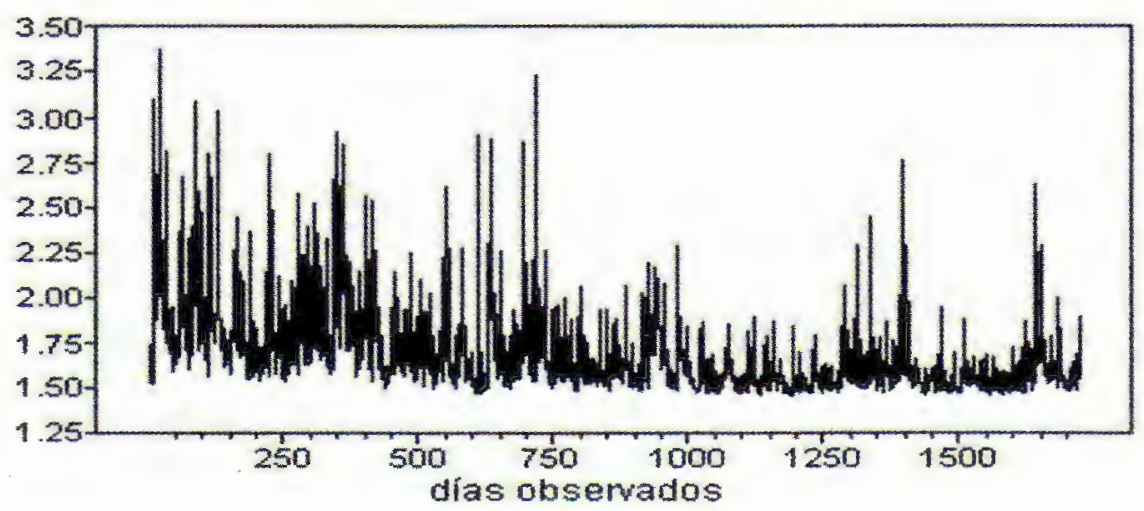

Gráfica 2.H Varios varianza condicional.

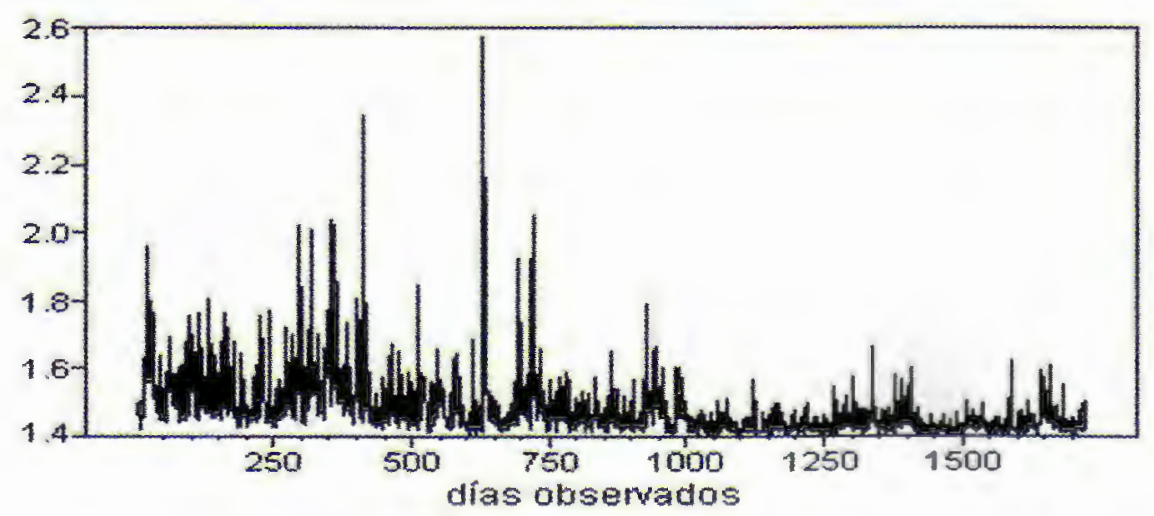


Se observa una disminución de los niveles de volatilidad para el periodo de auge en el mercado, tomaremos la fecha 2 enero 2003 (1054) como punto de corte, antes hay mayor volatilidad que después.

Por lo que los niveles de riesgo de los índices sectoriales y del IPC mostrados de mayor a menor riesgo son: Extractiva, Comunicaciones, Comercio, Servicios, IPC, Varios, Construcción, Transformación.

También es importante mencionar que se tienen los gråficos de covolatilidades del IPC con los sectores.

Gráfica 3.A Covolatilidad del IPC con Extractiva.

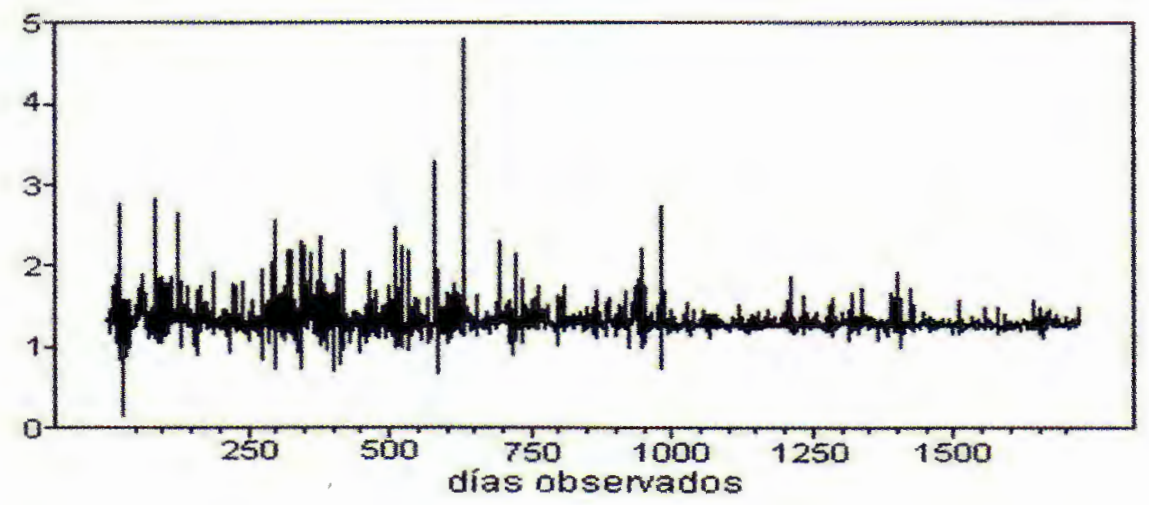

Gráfica 3.B Covolatilidad del IPC con Transformación.

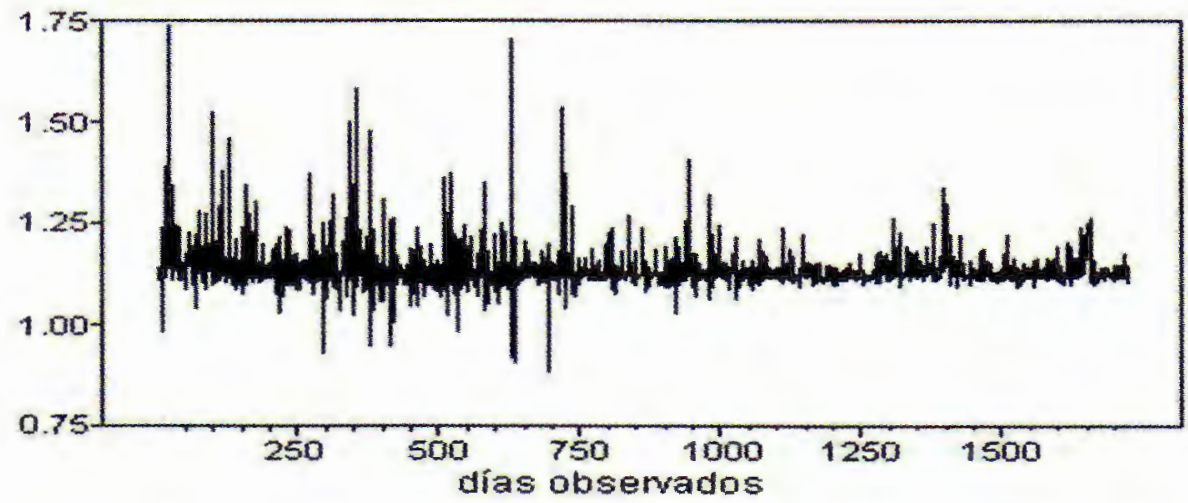




\section{Gráfica 3.C Covolatilidad de IPC con Construcción.}

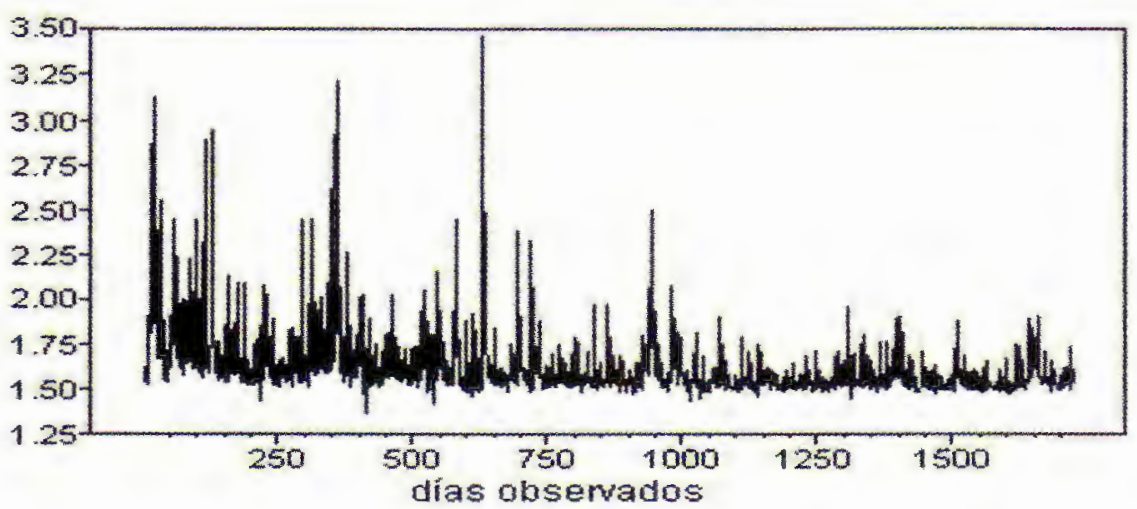

Gráfica 3.D Covolatilidad del IPC con Comercio.

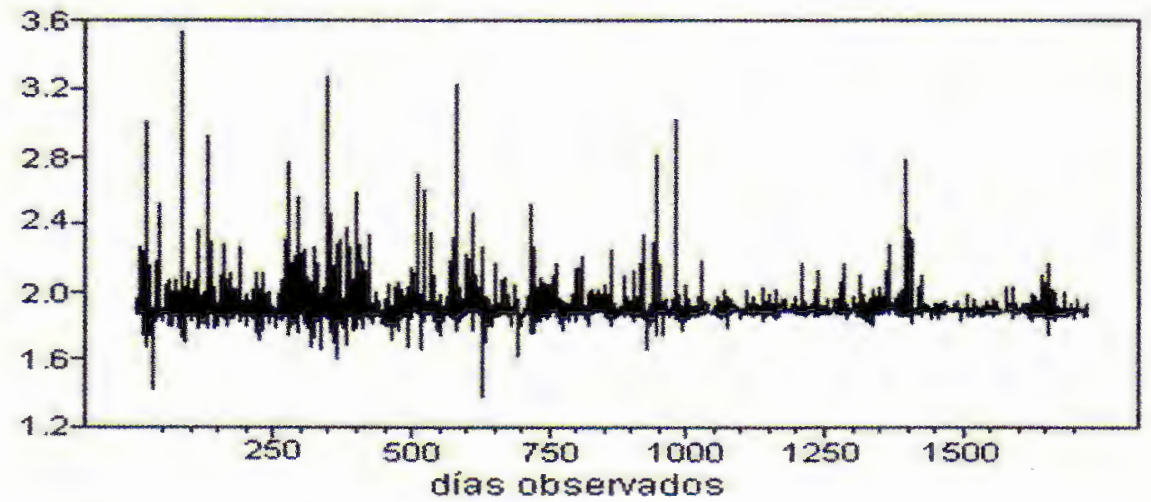

Gráfica 3.E Covolatilidad del IPC con Comunicaciones.

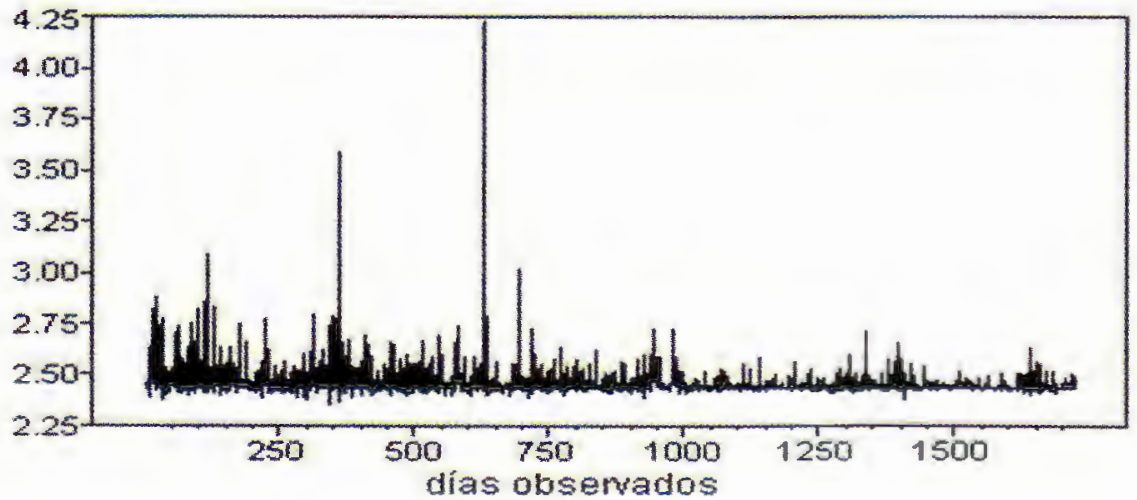


Gráfica 3.F Covolatilidad del IPC con Servicios.

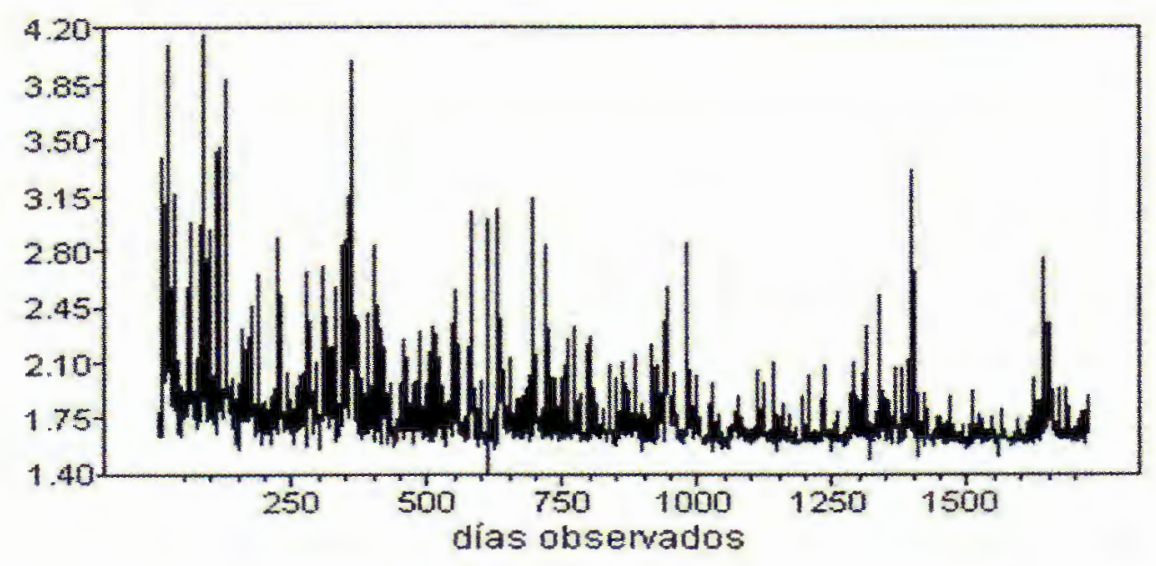

Gráfica 3.G Covolatilidad del IPC con Varios.

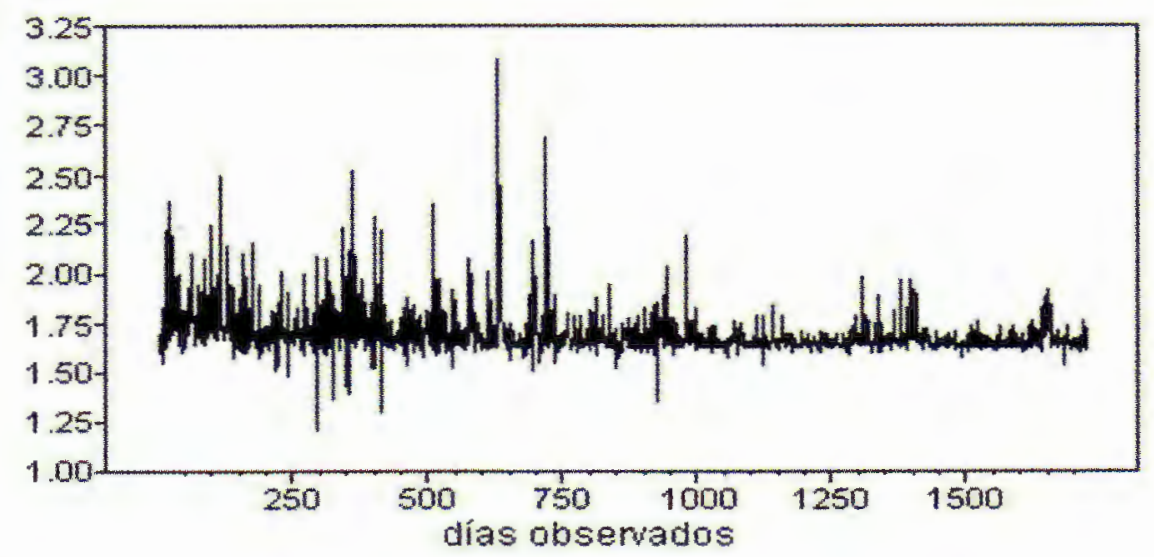

Estas covolatilidades están describiendo como los índices sectoriales se ajustan en relación al IPC, de modo que no debemos considerar la varianza condicional aislada sino además darnos cuenta de que los movimientos en la volatilidad son el "eco" de ajustes de las covolatilidades en el mercado, esta es una dirección en que debe darse más análisis para mejorar nuestra comprensión de la dinámica del riesgo.

\section{Conclusiones}

En este trabajo estudiamos la volatilidad de los rendimientos del IPC y de los siete índices sectoriales en el período (agosto 1998, julio 2005), enfatizando en los años 2003-2004 por los altos rendimientos reportados. Partimos de la teoría financiera de mercados de capital y específicamente de la HME y los modelos de equilibrio. En el mercado mexicano se corrobora la noción de que 
un mayor riesgo viene asociado con un mayor rendimiento, los cambios en la volatilidad de los rendimientos son persistentes en el tiempo en el sentido de que a mayor o menor volatilidad observada en un tiempo dado implica mayor o menor volatilidad para un intervalo de tiempo posterior. Si los choques en la volatilidad son persistentes, entonces los movimientos en los retornos requeridos también son persistentes, y el precio de equilibrio de los precios de los activos tiende a fluctuar más dramáticamente que lo que la mayoría de los modelos estándar predicen. ${ }^{4}$ Entonces, en el mercado mexicano se encontró evidencia del nivel de persistencia en la volatilidad de los rendimientos del IPC en el tiempo.

A través del estadístico $\operatorname{BDS}(m, \varepsilon)$ demostramos que la serie IPC, no es una serie IID sino que existe no linealidad en el comportamiento de los rendimientos. Modelamos la volatilidad a partir de $\mathrm{GARCH}(1,1)$ observamos que hay heterocedasticidad y correlación no serial. Además determinamos que se presenta covolatilidad, por lo que utilizamos el modelo GARCH Multivariado a partir de la formulación BEEK para capturar este efecto dinámico, el cual lo refleja exitosamente.

Finalmente, el estudio por los índices sectoriales nos deja con el deseo de ver la relación que existe entre el comportamiento del precio de las acciones y las variables macroeconómicas externas como el tipo de cambio, las tasas de interés o índices; cn este punto pensamos que el análisis de covolatilidad que se presenta entre los mercados es sin duda, un amplio tema de investigación.

\section{Anexo I}

Correspondencia entre las fechas y los números en las gráficas. Debido a que se manejan 1725 días operados, lo cual hace que las gráficas por fechas resulten totalmente ilegibles, decidimos establecer un consecutivo entre las fechas y los números en las gráficas; el cual se detalla a continuación: 21 de diciembre 1998 es el día 1; 2 de enero 2003 es el día 1054; 2 de enero 2004 es el día 1315; 2 de enero 2005 es el día 1598 y 29 de julio 2005 es el día 1725 .

\begin{tabular}{|cc|cc|cc|}
$21 / 12 / 1998$ & 1 & $19 / 06 / 2001$ & 652 & $16 / 12 / 2003$ & 1302 \\
$02 / 03 / 1999$ & 52 & $28 / 08 / 2001$ & 702 & $24 / 02 / 2004$ & 1352 \\
$11 / 05 / 1999$ & 102 & $06 / 11 / 2001$ & 752 & $04 / 05 / 2004$ & 1402 \\
$20 / 07 / 1999$ & 152 & $15 / 01 / 2002$ & 802 & $13 / 07 / 2004$ & 1452 \\
$28 / 09 / 1999$ & 202 & $26 / 03 / 2002$ & 852 & $21 / 09 / 2004$ & 1502 \\
$07 / 12 / 1999$ & 252 & $04 / 06 / 2002$ & 902 & $30 / 11 / 2004$ & 1552 \\
$15 / 02 / 2000$ & 302 & $13 / 08 / 2002$ & 952 & $08 / 02 / 2005$ & 1602 \\
$25 / 04 / 2000$ & 352 & $22 / 10 / 2002$ & 1002 & $19 / 04 / 2005$ & 1652 \\
$04 / 07 / 2000$ & 402 & $31 / 12 / 2002$ & 1052 & $28 / 06 / 2005$ & 1702 \\
$12 / 09 / 2000$ & 452 & $11 / 03 / 2003$ & 1102 & & \\
$21 / 11 / 2000$ & 502 & $20 / 05 / 2003$ & 1152 & & \\
$30 / 01 / 2001$ & 552 & $29 / 07 / 2003$ & 1202 & & \\
$10 / 04 / 2001$ & 602 & $07 / 10 / 2003$ & 1252 & &
\end{tabular}

4 Ver el trabajo de A. Poterba and L. Summers (1988). Mean Reversión in Stock Prices: Evidence and Implications. Journal of Finacial Economics, 22, 27-59. 


\section{Anexo II. Resultados de la estimación del modelo MV-GARCH(1,1)}

$\begin{array}{ccccc} & \text { Variable } & \text { Coeff. } & \text { Std. Error } & \text { T-Stat. } \\ 1 & \text { Mean(1) } & 0.041919 & 0.00328 & 12.77831 \\ 2 & \text { Mean(2) } & 0.070676 & 0.044541 & 1.58678 \\ 3 & \text { Mean(3) } & 0.009191 & 0.014699 & 0.62531 \\ 4 & \text { Mean(4) } & 0.058343 & 0.018763 & 3.10952 \\ 5 & \text { Mean(5) } & 0.049121 & 0.015591 & 3.15065 \\ 6 & \text { Mean(6) } & 0.046004 & 0.007155 & 6.42997 \\ 7 & \text { Mean(7) } & 0.077398 & 0.021202 & 3.65051 \\ 8 & \text { Mean(8) } & 0.014575 & 0.019323 & 0.75429 \\ 9 & \mathrm{C}(1,1) & 1.388197 & 0.00263 & 527.8706 \\ 10 & \mathrm{C}(2,1) & 0.919348 & 0.039554 & 23.24295 \\ 11 & \mathrm{C}(2,2) & 1.259121 & 0.030393 & 41.42842 \\ 12 & \mathrm{C}(3,1) & 0.786354 & 0.009444 & 83.26554 \\ 13 & \mathrm{C}(3,2) & 0.028278 & 0.012612 & -2.24219 \\ 14 & \mathrm{C}(3,3) & 0.275307 & 0.01319 & 20.87179 \\ 15 & \mathrm{C}(4,1) & 0.906843 & 0.012641 & 71.74098 \\ 16 & \mathrm{C}(4,2) & 0.019887 & 0.018353 & 1.08358 \\ 17 & \mathrm{C}(4,3) & 0.183256 & 0.01671 & 10.96709 \\ 18 & \mathrm{C}(4,4) & 0.584127 & 0.013602 & 42.94273 \\ 19 & \mathrm{C}(5,1) & 1.27543 & 0.011082 & 115.0894 \\ 20 & \mathrm{C}(5,2) & 0.154357 & 0.013772 & -11.2078 \\ 21 & \mathrm{C}(5,3) & 0.086855 & 0.014227 & 6.10506 \\ 22 & \mathrm{C}(5,4) & 0.249345 & 0.015135 & 16.47517 \\ 23 & \mathrm{C}(5,5) & 0.862838 & 0.011206 & 76.99913 \\ 24 & \mathrm{C}(6,1) & 1.605762 & 0.004649 & 345.4224 \\ 25 & \mathrm{C}(6,2) & 0.00246 & 0.007823 & 0.31449 \\ 26 & \mathrm{C}(6,3) & 0.165726 & 0.005746 & -28.8428 \\ 27 & \mathrm{C}(6,4) & 0.161991 & 0.005684 & -28.4977 \\ 28 & \mathrm{C}(6,5) & 0.406236 & 0.006488 & -62.6144 \\ 29 & \mathrm{C}(6,6) & 0.27573 & 0.004337 & 63.5834 \\ 30 & \mathrm{C}(7,1) & 1.074651 & 0.018869 & 56.95399 \\ 31 & \mathrm{C}(7,2) & 0.085139 & 0.021361 & -3.98575 \\ 32 & \mathrm{C}(7,3) & 0.191958 & 0.018367 & -10.4511 \\ 33 & \mathrm{C}(7,4) & 0.311726 & 0.018929 & -16.4684 \\ 34 & \mathrm{C}(7,5) & 0.280293 & 0.02088 & 13.42429 \\ 35 & \mathrm{C}(7,6) & 0.309504 & 0.026493 & -11.6824 \\ 36 & \mathrm{C}(7,7) & 0.312736 & 0.016345 & 19.13394 \\ 37 & \mathrm{C}(8,1) & 1.107857 & 0.014432 & 76.7625 \\ 38 & \mathrm{C}(8,2) & 0.137494 & 0.020842 & -6.59681 \\ 39 & \mathrm{C}(8,3) & 0.473819 & 0.017756 & 26.68491 \\ 40 & \mathrm{C}(8,4) & 0.255788 & 0.016575 & -15.4322 \\ 41 & \mathrm{C}(8,5) & 0.156631 & 0.017051 & 9.18591 \\ 42 & \mathrm{C}(8,6) & 0.100871 & 0.010171 & -9.91722 \\ 43 & \mathrm{C}(8,7) & 0.270726 & 0.013411 & -20.1874 \\ & & & & \end{array}$


Anexo II. (continuación)

\begin{tabular}{|c|c|c|c|}
\hline Variable & Coeff. & Std. Error & T-Stat. \\
\hline $\mathrm{C}(8,8)$ & 0.050887 & 0.018236 & 2.79039 \\
\hline $\mathrm{A}(1,1)$ & 0.079423 & 0.004008 & -19.8154 \\
\hline $\mathrm{A}(1,2)$ & 0.354346 & 0.04242 & 8.35331 \\
\hline$A(1,3)$ & 0.320047 & 0.017592 & 18.19282 \\
\hline $\mathrm{A}(1,4)$ & 0.091439 & 0.011834 & 7.72703 \\
\hline $\mathrm{A}(1,5)$ & 0.238372 & 0.021073 & -11.312 \\
\hline $\mathrm{A}(1,6)$ & 0.25089 & 0.008954 & 28.02092 \\
\hline $\mathrm{A}(1,7)$ & 0.652639 & 0.024966 & -26.1409 \\
\hline $\mathrm{A}(1,8)$ & 0.495121 & 0.02549 & 19.42447 \\
\hline$A(2,1)$ & 0.062705 & 0.003985 & 15.73461 \\
\hline $\mathrm{A}(2,2)$ & 0.051201 & 0.037954 & 1.34903 \\
\hline $\mathrm{A}(2,3)$ & 0.003026 & 0.011722 & -0.25815 \\
\hline $\mathrm{A}(2,4)$ & 0.033035 & 0.015788 & 2.09236 \\
\hline $\mathrm{A}(2,5)$ & 0.044891 & 0.015838 & 2.8344 \\
\hline $\mathrm{A}(2,6)$ & 0.025663 & 0.007465 & 3.43805 \\
\hline $\mathrm{A}(2,7)$ & 0.114408 & 0.019985 & 5.72479 \\
\hline $\mathrm{A}(2,8)$ & 0.024794 & 0.017485 & 1.41802 \\
\hline $\mathrm{A}(3,1)$ & 0.032579 & 0.004572 & 7.126 \\
\hline $\mathrm{A}(3,2)$ & 0.292841 & 0.07321 & -4.00004 \\
\hline $\mathrm{A}(3,3)$ & 0.024671 & 0.027184 & -0.90757 \\
\hline $\mathrm{A}(3,4)$ & 0.113928 & 0.025431 & -4.47986 \\
\hline $\mathrm{A}(3,5)$ & 0.065436 & 0.027438 & 2.38485 \\
\hline $\mathrm{A}(3,6)$ & 0.006696 & 0.01032 & -0.64885 \\
\hline $\mathrm{A}(3,7)$ & 0.683839 & 0.035732 & 19.13824 \\
\hline $\mathrm{A}(3,8)$ & 0.102713 & 0.040687 & -2.52449 \\
\hline $\mathrm{A}(4,1)$ & 0.058086 & 0.002755 & 21.08682 \\
\hline $\mathrm{A}(4,2)$ & 0.256062 & 0.050113 & -5.10965 \\
\hline $\mathrm{A}(4,3)$ & 0.022979 & 0.016572 & 1.3866 \\
\hline $\mathrm{A}(4,4)$ & 0.246445 & 0.0158 & 15.59826 \\
\hline $\mathrm{A}(4,5)$ & 0.069939 & 0.019466 & -3.59281 \\
\hline $\mathrm{A}(4,6)$ & 0.007829 & 0.006256 & 1.25146 \\
\hline $\mathrm{A}(4,7)$ & 0.034354 & 0.018032 & 1.90524 \\
\hline $\mathrm{A}(4,8)$ & 0.010851 & 0.025673 & 0.42268 \\
\hline $\mathrm{A}(5,1)$ & 0.048429 & 0.003531 & 13.71556 \\
\hline $\mathrm{A}(5,2)$ & 0.243775 & 0.051951 & 4.69243 \\
\hline$A(5,3)$ & 0.083135 & 0.017541 & -4.73947 \\
\hline $\mathrm{A}(5,4)$ & 0.017663 & 0.020833 & 0.84783 \\
\hline $\mathrm{A}(5,5)$ & 0.173601 & 0.019735 & 8.7965 \\
\hline $\mathrm{A}(5,6)$ & 0.033802 & 0.007979 & -4.23621 \\
\hline $\mathrm{A}(5,7)$ & 0.237771 & 0.021889 & 10.86236 \\
\hline $\mathrm{A}(5,8)$ & 0.234781 & 0.023216 & -10.1131 \\
\hline $\mathrm{A}(6,1)$ & 0.002729 & 0.002318 & 21.17733 \\
\hline $\mathrm{A}(6,2)$ & 0.023799 & 0.037183 & 0.64006 \\
\hline
\end{tabular}


Anexo II. (continuación)

$\begin{array}{ccccc} & \text { Variable } & \text { Coeff. } & \text { St.d. Error } & \text { T-Stat. } \\ 87 & \mathrm{~A}(6,3) & 0.08457 & 0.013171 & -6.42089 \\ 88 & \mathrm{~A}(6,4) & 0.043071 & 0.009837 & -4.37833 \\ 89 & \mathrm{~A}(6,5) & 0.171972 & 0.013885 & 12.38555 \\ 90 & \mathrm{~A}(6,6) & 0.164595 & 0.007671 & -21.458 \\ 91 & \mathrm{~A}(6,7) & 0.094139 & 0.017046 & 5.52263 \\ 92 & \mathrm{~A}(6,8) & 0.24764 & 0.020674 & -11.9782 \\ 93 & \mathrm{~A}(7,1) & 0.056477 & 0.002115 & 26.70184 \\ 94 & \mathrm{~A}(7,2) & 0.11556 & 0.035344 & 3.26959 \\ 95 & \mathrm{~A}(7,3) & 0.032448 & 0.012444 & -2.6075 \\ 96 & \mathrm{~A}(7,4) & 0.098222 & 0.009304 & 10.55663 \\ 97 & \mathrm{~A}(7,5) & 0.035816 & 0.013477 & -2.6575 \\ 98 & \mathrm{~A}(7,6) & 0.089439 & 0.004886 & 18.30509 \\ 99 & \mathrm{~A}(7,7) & 0.229239 & 0.015771 & 14.5355 \\ 100 & \mathrm{~A}(7,8) & 0.020865 & 0.02025 & -1.03036 \\ 101 & \mathrm{~A}(8,1) & 0.02985 & 0.003698 & -8.07255 \\ 102 & \mathrm{~A}(8,2) & 0.115574 & 0.052176 & -2.21509 \\ 103 & \mathrm{~A}(8,3) & 0.046656 & 0.018213 & -2.56172 \\ 104 & \mathrm{~A}(8,4) & 0.144863 & 0.016593 & -8.73059 \\ 105 & \mathrm{~A}(8,5) & 0.16434 & 0.017321 & 9.48772 \\ 106 & \mathrm{~A}(8,6) & 0.118576 & 0.00863 & -13.7402 \\ 107 & \mathrm{~A}(8,7) & 0.153802 & 0.018988 & -8.10009 \\ 108 & \mathrm{~A}(8,8) & 0.131993 & 0.026099 & 5.05739 \\ 109 & \mathrm{~B}(1,1) & 0.040522 & 0.00178 & 22.7655 \\ 110 & \mathrm{~B}(1,2) & 0.080048 & 0.041823 & 1.91397 \\ 111 & \mathrm{~B}(1,3) & 0.095232 & 0.011053 & 8.6159 \\ 112 & \mathrm{~B}(1,4) & 0.184844 & 0.014863 & -12.4363 \\ 113 & \mathrm{~B}(1,5) & 0.038413 & 0.010358 & 3.70842 \\ 114 & \mathrm{~B}(1,6) & 0.0362 & 0.004105 & 8.81908 \\ 115 & \mathrm{~B}(1,7) & 0.103356 & 0.013938 & -7.4154 \\ 116 & \mathrm{~B}(1,8) & 0.524025 & 0.022691 & -23.0942 \\ 117 & \mathrm{~B}(2,1) & 0.051945 & 0.002303 & 22.55154 \\ 118 & \mathrm{~B}(2,2) & 0.014367 & 0.037394 & 0.38419 \\ 119 & \mathrm{~B}(2,3) & 0.146244 & 0.009388 & -15.5771 \\ 120 & \mathrm{~B}(2,4) & 0.133961 & 0.014219 & 9.42139 \\ 121 & \mathrm{~B}(2,5) & 0.005832 & 0.011435 & -0.50998 \\ 122 & \mathrm{~B}(2,6) & 0.111671 & 0.004598 & 24.28659 \\ 123 & \mathrm{~B}(2,7) & 0.115526 & 0.015205 & -7.59784 \\ 124 & \mathrm{~B}(2,8) & 0.235696 & 0.016284 & 14.47432 \\ 125 & \mathrm{~B}(3,1) & 0.02626 & 0.00276 & 9.51327 \\ 126 & \mathrm{~B}(3,2) & 0.118388 & 0.066135 & 1.7901 \\ 127 & \mathrm{~B}(3,3) & 0.34966 & 0.016808 & 20.80346 \\ 128 & \mathrm{~B}(3,4) & 0.269401 & 0.021613 & -12.4645 \\ 129 & \mathrm{~B}(3,5) & 0.241762 & 0.016972 & 14.24484 \\ & & & & \\ & & & \end{array}$


Anexo II. (continuación)

\begin{tabular}{|c|c|c|c|c|}
\hline & Variable & Coeff. & Std. Error & T-Stat. \\
\hline 130 & $\mathrm{~B}(3,6)$ & 0.050783 & 0.006242 & 8.13519 \\
\hline 131 & $\mathrm{~B}(3,7)$ & 0.359315 & 0.026944 & -13.3354 \\
\hline 132 & $\mathrm{~B}(3,8)$ & 0.08587 & 0.032091 & 2.67585 \\
\hline 133 & $\mathrm{~B}(4,1)$ & 0.046391 & 0.002256 & 20.56417 \\
\hline 134 & $\mathrm{~B}(4,2)$ & 0.113149 & 0.047102 & -2.40219 \\
\hline 135 & $\mathrm{~B}(4,3)$ & 0.2317 & 0.0123 & -18.8367 \\
\hline 136 & $\mathrm{~B}(4,4)$ & 0.065563 & 0.015426 & 4.25015 \\
\hline 137 & $\mathrm{~B}(4,5)$ & 0.010479 & 0.012326 & 0.85019 \\
\hline 138 & $\mathrm{~B}(4,6)$ & 0.077734 & 0.004687 & 16.58558 \\
\hline 139 & $\mathrm{~B}(4,7)$ & 0.629982 & 0.019332 & 32.58709 \\
\hline 140 & $\mathrm{~B}(4,8)$ & 0.181018 & 0.018306 & 9.88 \\
\hline 141 & $\mathrm{~B}(5,1)$ & 0.024527 & 0.002037 & 12.04333 \\
\hline 142 & $\mathrm{~B}(5,2)$ & 1.132458 & 0.044223 & -25.608 \\
\hline 143 & $\mathrm{~B}(5,3)$ & 0.125219 & 0.011842 & -10.8 \\
\hline 144 & $\mathrm{~B}(5,4)$ & 0.087462 & 0.014812 & -5.90486 \\
\hline 145 & $\mathrm{~B}(5,5)$ & 0.085818 & 0.010881 & 7.88687 \\
\hline 146 & $\mathrm{~B}(5,6)$ & 0.172885 & 0.006654 & 25.98271 \\
\hline 147 & $\mathrm{~B}(5,7)$ & 0.083495 & 0.015184 & -5.49884 \\
\hline 148 & $\mathrm{~B}(5,8)$ & 0.14254 & 0.018791 & -7.58568 \\
\hline 149 & $\mathrm{~B}(6,1)$ & 0.030715 & 0.001726 & 17.79854 \\
\hline 150 & $\mathrm{~B}(6,2)$ & 0.686064 & 0.04177 & 16.42465 \\
\hline 151 & $\mathrm{~B}(6,3)$ & 0.051043 & 0.009983 & -5.11292 \\
\hline 152 & $\mathrm{~B}(6,4)$ & 0.026084 & 0.012861 & -2.02812 \\
\hline 153 & $\mathrm{~B}(6,5)$ & 0.152336 & 0.01035 & 14.71841 \\
\hline 154 & $\mathrm{~B}(6,6)$ & 0.019556 & 0.003806 & 5.13842 \\
\hline 155 & $\mathrm{~B}(6,7)$ & 0.146979 & 0.012967 & -11.3345 \\
\hline 156 & $\mathrm{~B}(6,8)$ & 0.257117 & 0.01622 & 15.85175 \\
\hline 157 & $\mathrm{~B}(7,1)$ & 0.085495 & 0.00255 & 33.52198 \\
\hline 158 & $\mathrm{~B}(7,2)$ & 0.166692 & 0.043003 & 3.87628 \\
\hline 159 & $\mathrm{~B}(7,3)$ & 0.219374 & 0.01284 & 17.08485 \\
\hline 160 & $\mathrm{~B}(7,4)$ & 0.453755 & 0.015142 & 29.96569 \\
\hline 161 & $\mathrm{~B}(7,5)$ & 0.199311 & 0.012695 & -15.7001 \\
\hline 162 & $\mathrm{~B}(7,6)$ & 0.11496 & 0.006513 & -17.652 \\
\hline 163 & $\mathrm{~B}(7,7)$ & 0.419492 & 0.017949 & 23.37171 \\
\hline 164 & $\mathrm{~B}(7,8)$ & 0.340167 & 0.017978 & 18.92156 \\
\hline 165 & $\mathrm{~B}(8,1)$ & 0.020632 & 0.002048 & 10.07195 \\
\hline 166 & $\mathrm{~B}(8,2)$ & 0.048606 & 0.046699 & 1.04085 \\
\hline 167 & $\mathrm{~B}(8,3)$ & 0.048708 & 0.015111 & 3.22344 \\
\hline 168 & $\mathrm{~B}(8,4)$ & 0.377078 & 0.023132 & 16.301 \\
\hline 169 & $\mathrm{~B}(8,5)$ & 0.044165 & 0.012474 & -3.54059 \\
\hline 170 & $\mathrm{~B}(8,6)$ & 0.080956 & 0.00458 & 17.67738 \\
\hline 171 & $\mathrm{~B}(8,7)$ & 0.017905 & 0.015337 & 1.16744 \\
\hline 172 & $\mathrm{~B}(8,8)$ & 0.192131 & 0.017813 & -10.7861 \\
\hline
\end{tabular}




\section{Bibliografía}

Abhyankar, A., L. Copeland, and W. Wong (1997). Uncovering Nonlinear Structure in Real-Time Stock-Market Indexes: The S\&P 500, the DAX, the Nikkei 225, and the FTSE-100. Journal of Business \& Economic Statistics, 15, pp. 1-14.

Black, F and M. Sholes (1973). The Valuation of Options and Corporate Liabilities. Journal. of Political Economy, 81, pp. 637-59.

Bollerslev, T. (1986). Generalized Autoregressive Conditional Heteroscedasticity. Journal of Econometrics, 31, pp. 307-327.

Chan, K. C. and N. Chen (1991). Structural and Return characteristics of small and large firms. Journal of Finance, 46, pp. 1467-1484.

Chen, N., R. Roll, and S. Ross (1986). Economic Forces and the Stock Market. Journal of Business, 59, pp. 383-403.

Chopra, N., J. Lakonishok, and J. Ritter (1992). Measuring Abnormal Performance: Do Stocks Overreact. Journal of Finance.

DeBondt, W. and R. Thaler (1985). Does the Stock Market Overreact. Journal of Finance, 40, pp. 793-805.

Engle, R. (1982). Autorregresive Conditional Heteroscedasticity whit Estimates of the Variance of United Kingdom Inflations. Econometrica, 50, pp. 987-1008.

Fama, E. (1970). Efficient Capital Markets: A Review of Theory and Empirical Work. Journal of Finance, pp. 383-417.

Fama E. (1991). Efficient Capital Markets: II. Journal of Finance, 46, pp. 575-1617.

Fama, E. and R. Roll (1969). The Adjustment of Stock Prices to New Information. International Economic Review, 10(1), pp. 1-21.

Fama, E. and F. Kenneth (1988), Permanent and Temporary Components of Stock Prices, Journal of Political Economy.

Fama, E. and F. Kenneth (1992). The Cross-Section of Expected Stock Returns. Journal of Finance, 47, pp. 427-465.

Fama, E. and F. Kenneth (1993). Multifactor Explanations of Asset Pricing Anomaliaes. Journal of Finance, 51(1), pp. 55-83.

Fama, E. and J. MacBeth (1973). Risk, Return and Equilibrium: Empirical Test. Jourral of Political Economy, pp. 607-636.

Jegadeesh, N. and S. Titman (1993). Returns to buying winners and sellings losers: Implications for stock market efficient. Journal of Finance, 48, pp. 65-91.

Kwon, C. and F. Bacon (1997). The Effect of Macroeconomic variables on Stock Market Returns in developing markets. Multinational Business Review.

Ludlow-Wiechers, J.' (2005). Contagio financiero, volatilidades y covolatilidades entre los mercados latinoamericanos. Por aparecer.

Mandelbrot, B. (1966). Forecasts of Future Prices, Unbiased Markets, and Martingale Models. Journal of Business, 39, pp. 242-255.

Peters, P. (2001). Estimating y Forecasting Volatility of Stock Indices using Asymmetric GARCH models y (Skewed) Student-t densities. Mimeo, Université de Liége, Bélgica, $23(20)$.

Poterba, A and L. Summers (1988). Mean Reversion in Stock Prices: Evidence and Implications. Journal of Finacial Economics, 22, pp. 27-59.

Samuelson, P. (1965). Proof That Properly Anticipated Prices Fluctuase Randomly. Industrial Management Review, pp. 41-49. 